\title{
THE
}

\section{Three-Dimensional Kinematics and Wake Structure of the Pectoral Fins During Locomotion in Leopard Sharks Triakis semifasciata}

\author{
C. D. Wilga \\ University of Rhode Island, cwilga@uri.edu \\ George V. Lauder
}

Follow this and additional works at: https://digitalcommons.uri.edu/bio_facpubs

Terms of Use

All rights reserved under copyright.

\section{Citation/Publisher Attribution}

Wilga, C. D. and G. V. Laudar. "Three-Dimensional Kinematics and Wake Structure of the Pectoral Fins During Locomotion in Leopard Sharks Triakis semifasciata." Journal of Experimental Biology. 203(15):2261-2278. August 2000.

Available at http://jeb.biologists.org/content/203/15/2261.full.pdf+html JEB2774

This Article is brought to you for free and open access by the Biological Sciences at DigitalCommons@URI. It has been accepted for inclusion in Biological Sciences Faculty Publications by an authorized administrator of DigitalCommons@URI. For more information, please contact digitalcommons-group@uri.edu. 


\title{
THREE-DIMENSIONAL KINEMATICS AND WAKE STRUCTURE OF THE PECTORAL FINS DURING LOCOMOTION IN LEOPARD SHARKS TRIAKIS SEMIFASCIATA
}

\author{
C. D. WILGA*, $\$$ AND G. V. LAUDER $\$$ \\ Department of Ecology and Evolutionary Biology, University of California at Irvine, Irvine, CA 92697, USA \\ *e-mail: cwilga@oeb.harvard.edu \\ \$resent address: Museum of Comparative Zoology, Harvard University, 26 Oxford Street, Cambridge, MA 02138, USA
}

Accepted 10 May; published on WWW 10 July 2000

\begin{abstract}
Summary
The classical theory of locomotion in sharks proposes that shark pectoral fins are oriented to generate lift forces that balance the moment produced by the oscillating heterocercal tail. Accordingly, previous studies of shark locomotion have used fixed-wing aircraft as a model assuming that sharks have similar stability and control mechanisms. However, unlike airplanes, sharks are propelled by undulations of the body and tail and have considerable control of pectoral fin motion. In this paper, we use a new approach to examine the function of the pectoral fins of leopard sharks, Triakis semifasciata, during steady horizontal swimming at speeds of $0.5-2.0 l \mathrm{~s}^{-1}$, where $l$ is total body length, and during vertical maneuvering (rising and sinking) in the water column. The planar orientation of the pectoral fin was measured using threedimensional kinematics, while fluid flow in the wake of the pectoral fin and forces exerted on the water by the fin were quantified using digital particle image velocimetry (DPIV). Steady horizontal swimming in leopard sharks is characterized by continuous undulations of the body with a positive body tilt to the flow that decreases from a mean of $11^{\circ}$ to $0.6^{\circ}$ with increasing flow speeds from 0.5 to

Leopard shark pectoral fins are also oriented at a substantial negative dihedral angle that amplifies roll moments and hence promotes rapid changes in body position. Vortices shed from the trailing edge of the pectoral fin were detected only during vertical maneuvering. Starting vortices are produced when the posterior plane of the pectoral fin is actively flipped upwards or downwards to initiate rising or sinking, respectively, in the water column. The starting vortex produced by the pectoral fin induces a pitching moment that reorients the body relative to the flow. Body and pectoral fin surface angle are altered significantly when leopard sharks change vertical position in the water column. Thus, locomotion in leopard sharks is not analogous to flight in fixed-wing aircraft. Instead, a new force balance for swimming leopard sharks is proposed for steady swimming and maneuvering. Total force balance on the body is adjusted by altering the body angle during steady swimming as well as during vertical maneuvering, while the pectoral fins appear to be critical for initiating maneuvering behaviors, but not for lift production during steady horizontal locomotion.
\end{abstract} $2.0 l \mathrm{~s}^{-1}$. Three-dimensional analysis showed that, during steady horizontal locomotion, the pectoral fins are cambered, concave downwards, at a negative angle of attack that we predict to generate no significant lift.
Key words: leopard shark, locomotion, pectoral fin, flow visualization, force balance, hydrodynamics, Triakis semifasciata.

\section{Introduction}

According to the classical view of locomotor biomechanics in sharks (Affleck, 1950; Alexander, 1965; Simons, 1970; Thomson, 1976; Videler, 1993), the pectoral fins function to generate lift forces anterior to the center of mass and hence generate torque that counters the torque introduced by the heterocercal tail. Because of its morphological asymmetry in the horizontal plane, the heterocercal tail of sharks has long been thought to generate forces at an angle to the body axis, in contrast to the function of the homocercal tail in teleost fishes (Lauder, 1989). Recently, the tail component of the classical theory of shark locomotion has been corroborated in a study of freely swimming leopard sharks Triakis semifasciata
(Ferry and Lauder, 1996). Using three-dimensional video analysis and dye-stream visualization, these authors proposed that movement of the tail surface deflects water ventrally and posteriorly, generating an anterodorsally directed reaction force with both lift and thrust components.

While further research on tail function is certainly needed to determine the generality of this result in other shark species, pectoral fin function hypothesized by the classical model of shark locomotion has not yet been examined quantitatively. However, evidence based on manipulation of living sharks and studies of mechanical models has supported the view that the pectoral fins provide lift in swimming sharks. Daniel (1922) 
disabled the pectoral fins of sharks and found that, after releasing them into the water, they were unable to swim steadily and headed downwards towards the substratum. Harris (1936) conducted wind-tunnel experiments on a model of Mustelus canis in which the pectoral fins were set at an angle of inclination to the body axis of $8-10^{\circ}$ and found that the presence of the pectoral fins greatly increased pitching moments. Several studies have also proposed that sharks turn and maneuver by altering the angle of the pectoral fins as well as the pectoral fin surface (Daniel, 1922; Harris, 1936; Klausewitz, 1962, 1965; Simons, 1970; Thomson and Simanek, 1977), although others suggest that the pectoral fins are relatively fixed in position and move little (Breder, 1926). Fish and Shannahan (2000) videotaped sharks swimming in a public aquarium and concluded that the pectoral fins are oriented in such a way as to generate lift.

Given the paucity of empirical data on the function of the pectoral fins during locomotion in sharks, our main objective here is to test hypothesized functions of the pectoral fins under the classical theory of shark locomotion by studying freely swimming sharks of the same species (Triakis semifasciata, leopard sharks) used by Ferry and Lauder (1996) in their analysis of tail function. We test this hypothesis in two ways. First, we quantify the three-dimensional kinematic positions of the pectoral fins during steady horizontal swimming as well as during rising and sinking maneuvering behaviors to determine whether the pectoral fins are held in an orientation that would be expected to generate lift. Second, we quantify water flow in the wake of the pectoral fins during locomotion in leopard sharks using digital particle image velocimetry (DPIV) (Willert and Gharib, 1991; Krothapalli et al., 1997), which allows estimates of fluid vorticity and, hence, the forces exerted by the fin on the fluid (see Drucker and Lauder, 1999; Wilga and Lauder, 1999). Our secondary objective is to compare these data on leopard sharks with our previous results on locomotion in the white sturgeon Acipenser transmontanus, a ray-finned fish species also possessing a heterocercal tail (Wilga and Lauder, 1999).

\section{Materials and methods Animals}

Leopard sharks, Triakis semifasciata Girard, were obtained from Santa Monica Bay off Southern California under Scientific Permit number 801133-4 from the State of California. Sharks were housed in 13251 aquaria at a temperature of $20 \pm 2{ }^{\circ} \mathrm{C}$ and maintained on a diet of smelt. Experiments were conducted in a calibrated flow tank maintained at an average temperature of $20 \pm 1^{\circ} \mathrm{C}$ (see Jayne and Lauder, 1995; Jayne et al., 1996; Wilga and Lauder, 1999). Four individuals averaging $44 \mathrm{~cm}$ in total length $(l)$ (range $39-47 \mathrm{~cm}$ ) were used for the kinematic and digital particle image velocimetry (DPIV) experiments described below.

\section{Kinematics}

Kinematic methods for sharks followed those used for our previous paper on sturgeon (Wilga and Lauder, 1999) as closely as possible so that data could be compared between species. Briefly, sharks were videotaped using a NAC HSV500 high-speed video system at 250 fields s $^{-1}$ (resolution 640 pixels $\times 480$ pixels) while swimming in a 6001 flow tank with a working area of $82 \mathrm{~cm}$ long $\times 28 \mathrm{~cm}$ wide $\times 28 \mathrm{~cm}$ high. A lateral view of the swimming sharks was recorded by directing one camera perpendicular to the side of the flow tank, while the ventral view was recorded by aiming a second camera at a front-surface mirror oriented at $45^{\circ}$ below the working section of the tank. For all experimental protocols, the two cameras were electronically synchronized. The filming distance was large enough for parallax errors to be negligible. Marked grids were used to scale lateral and ventral recordings similarly. Whole body (zoomed-out) views were recorded to analyze speed and behavior effects on body angle, while head and pectoral (zoomed-in) views were recorded for detailed three-dimensional analysis of pectoral fin conformation. A custom-designed computer program was used to digitize video sequences.

Four swimming speeds, $0.5, \quad 1.0, \quad 1.5 \quad$ and 2.0 total body lengths $\mathrm{s}^{-1} \quad\left(l \mathrm{~s}^{-1}\right)$, while fish were holding position in the flow were examined to investigate whether sharks alter their body angle with swimming speed. Each individual was filmed swimming at four speeds using the whole body camera arrangement. In total, 400 images were digitized for the speed effect experiments: five fields evenly spaced within each tailbeat for five different tailbeat sequences at four speeds in each of four individuals.

Holding position, rising and sinking in the water column were studied as the sharks swam in the flow tank at $1.0 l \mathrm{~s}^{-1}$. We investigated whether sharks are capable of altering the angle of the body and pectoral fins to change position in the water column using both the whole body and the head and pectoral fin camera arrangements as described above. Holding position is defined as the animal maintaining a stationary (within $2 \% l \mathrm{~s}^{-1}$ deviation from a fixed frame of reference) horizontal (anteroposterior) and vertical position in the water column. We define rising and sinking as maintaining horizontal position in the water column while actively increasing or decreasing vertical position by at least $4 \mathrm{~cm} \mathrm{~s}^{-1}$ with minimal lateral movement. Only those video sequences were analyzed in which the shark maintained horizontal and vertical position during holding or ascended or descended smoothly in the water column during rising and sinking, in all cases with minimal lateral, upstream-downstream, pitching (except when changing vertical position) or roll motions (within $2 \% l \mathrm{~s}^{-1}$ deviation from a fixed frame of reference). Pitching movements are necessary when rising and sinking behaviors are initiated. To test whether sharks are capable of altering pectoral fin movements with behavior, holding, rising and sinking sequences were chosen a priori from videotape recordings. Only sequences in which any body or pectoral fin surface of the shark was at least $4 \mathrm{~cm}$ from any side of the flow tank or the surface of the water were analyzed to minimize potential boundary effects from the tank walls on the flow 
Fig. 1. Schematic diagram of a leopard shark Triakis semifasciata illustrating the digitized points on the body and pectoral fin. (A) Lateral view showing the entire body; (B) lateral view of the head and pectoral fin; (C) ventral view of pectoral fin region. Note that the reference axes differ for lateral $(x, y)$ and ventral $(x, z)$ views. Data in $\mathrm{B}$ and $\mathrm{C}$ were recorded simultaneously, while data in A were obtained separately using a different magnification so that the entire animal was visible. Therefore, homologous points in A and $\mathrm{B}$ are numbered differently to reflect points that were not digitized from images recorded concurrently and at equivalent magnification. Points $14-16$ are the same points in lateral and ventral views, while points 17 and $17 \mathrm{v}$ represent the same

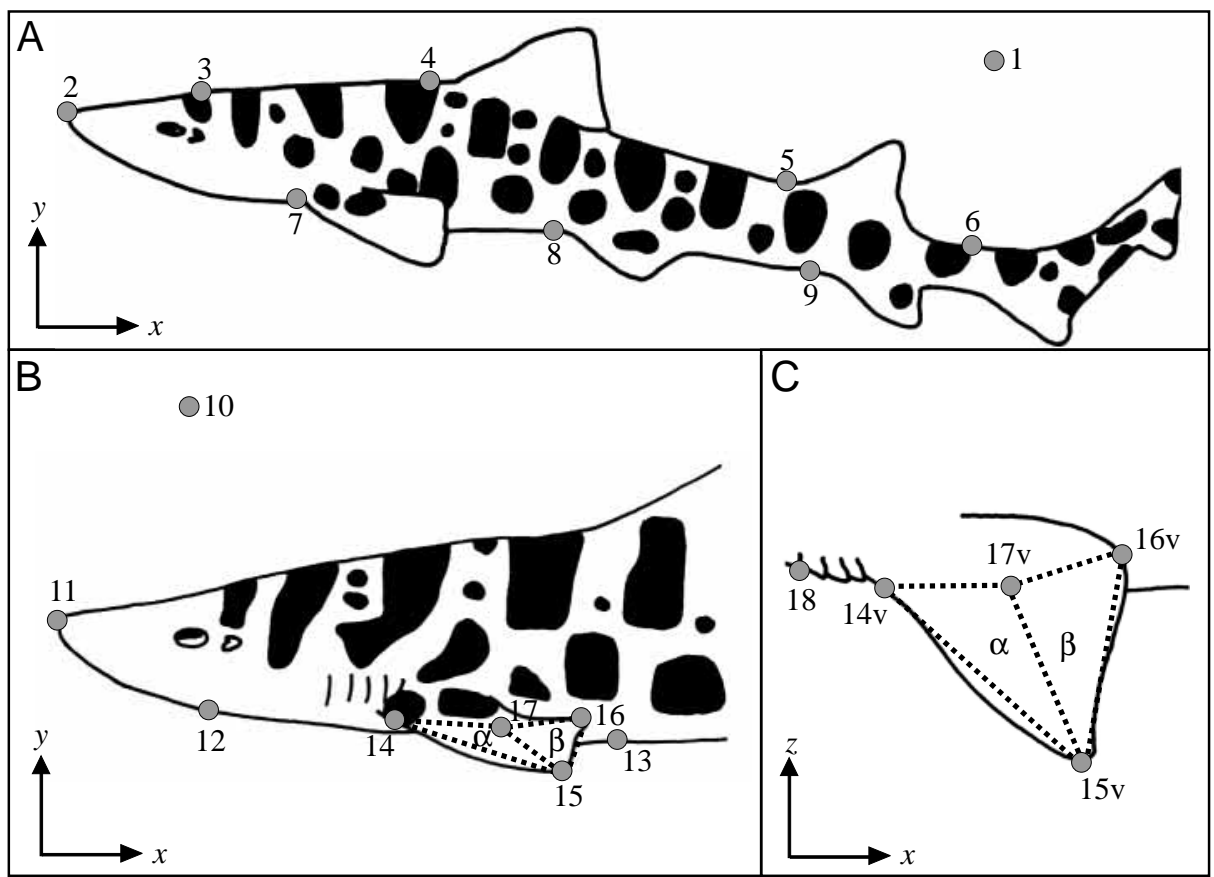
location on the dorsal and ventral fin surfaces. These three-dimensional coordinate data were used to calculate a three-dimensional planar angle between the anterior and posterior fin planes ( $\alpha$ and $\beta$ ) as shown in B and C.

around the fish for all experimental protocols (Webb, 1993). In total, 300 digitized images for the whole body experiments were analyzed for the behavior experiments: five fields equally spaced throughout a tailbeat for five tailbeats in four individuals for three behaviors.

On lateral views of the whole body (Fig. 1A) for the speed effect and behavioral analyses, the $x$ (horizontal) and $y$ (vertical) coordinates of nine points were digitized: point 1 , fixed reference mark on the background; 2, snout tip; 3 , posterodorsal point of first dorsal pigment mark; 4, posterodorsal point of fourth dorsal pigment mark just anterior to dorsal fin; 5 , anterior base of second dorsal fin; 6 , dorsal caudal peduncle; 7, anterior base of pectoral fin; 8, anterior base of pelvic fin; 9, anterior base of anal fin.

On simultaneous lateral and ventral views of the head and pectoral fins (Fig. 1B,C), the $x, y$ and $z$ coordinates of 11 points were digitized: point 10, fixed reference mark on the background; 11, snout tip; 12, ventral body surface ventral to posterior margin of the eye; 13 , ventral body surface at anterior base of first dorsal fin; 14, anterior base of pectoral fin; 15, lateral pectoral fin tip; 16, posterior edge of pectoral fin; 17 , internal marked location on fin surface. On ventral images of the pectoral fins, the $x$ and $z$ coordinates of six points were digitized (Fig. 1C): $14 \mathrm{v}$ anterior base of pectoral fin; 15v, lateral fin tip; 16v, posterior fin margin; $17 \mathrm{v}$, medial marked point on the fin; 18 , first gill slit.

Points $14-17$ on the shark pectoral fins were marked to provide reliable landmarks for video digitizing prior to filming the head and pectoral (zoomed-in) views. Preliminary video recordings revealed that reliable identification of specific points on the fin margins and interior was not possible without marking the fins. To this end, sharks were anesthetized using $0.15 \mathrm{gl}^{-1}$ tricaine methanesulfate (MS-222), intubated and maintained on $0.065 \mathrm{~g}^{-1}$ MS-222 while reflective aluminum markers were glued to the pectoral fins using cyanoacrylate adhesive. Rectangular markers $(0.5 \mathrm{~cm} \times 0.2 \mathrm{~cm})$ for points $14-16$ and $14 \mathrm{v}-16 \mathrm{v}$ were wrapped around the edge of the fin so that they were visible in both lateral and ventral images. Small square markers $\left(0.2 \mathrm{~cm}^{2}\right)$ for points 17 and $17 \mathrm{v}$ were glued to the dorsal and ventral surfaces of the fin. Preliminary video recordings were used to place points 17 and $17 \mathrm{v}$ at a position where the posterior portion of the fin begins to move vertically (as a flap) during rising and sinking behaviors. After the markers had been glued in place, the sharks were intubated with fresh water until swimming movements began, after which they were returned to the flow tank and allowed to recover for at least $3 \mathrm{~h}$ prior to video recording.

As in our previous paper (Wilga and Lauder, 1999), a threedimensional planar angle between the two triangles comprising the pectoral fin (Fig. 1: $\alpha$ and $\beta$ ) (in the animal frame of reference) was calculated in addition to the angle that each of these triangles makes with three perpendicular reference planes in the earth frame of reference. These angles were used to detect whether the position and conformation of the pectoral fin surface is altered among the three behaviors during locomotion. Examination of video sequences and digitized data revealed that movement of the posterior region of the pectoral fin is well represented by plane $\beta$, and that movement of the entire surface of the pectoral fin is well-represented by quantifying the position of the two planes $\alpha$ and $\beta$.

The working space of the flow tank in which sharks moved can be divided into a standard Cartesian coordinate system in 
which the position of any point in space can be identified by $x, y$ and $z$ values (Fig. 1). The measurements and calculations follow the procedures used in previous research (Ferry and Lauder, 1996; Lauder and Jayne, 1996; Wilga and Lauder, 1999). The lower left-hand corner in the lateral view for the $x$ (horizontal) and $y$ (vertical) dimensions and the lower left-hand corner in the ventral view for the $x$ (horizontal) and $z$ (vertical) dimensions was defined as the origin of the coordinate system. The $x, y$ and $z$ coordinates for each point were obtained by using the $x$ and $y$ coordinates in horizontal view and the $z$ coordinate in ventral view, the redundant $x$ coordinate data in the ventral recording was deleted from the data. Points $14,14 \mathrm{v}$, $15,15 \mathrm{v}, 17$ and $17 \mathrm{v}$ were used to form fin triangle $\alpha$ and points $15,15 \mathrm{v}, 16,16 \mathrm{v}, 17$ and $17 \mathrm{v}$ were used to form fin triangle $\beta$. The angle that triangles $\alpha$ and $\beta$ make with the three orthogonal reference planes, parasagittal $(X Y)$, transverse $(Y Z)$ and frontal $(X Z)$, was calculated by determining the orientation of the triangles in three-dimensional space. The parasagittal plane is parallel to the longitudinal axis of the animal when swimming and is equivalent to the side of the flow tank (visible as the lateral camera view). The transverse plane is the plane towards which the shark is swimming and is represented as the front wall of the flow tank. The frontal plane is equivalent to the floor of the tank (visible as the ventral view). The planar angle of intersection between fin triangles $\alpha$ and $\beta$ with each of the three reference planes was calculated from the $x, y$ and $z$ coordinates.

\section{Digital particle image velocimetry}

Water flow in the wake of the pectoral fins of sharks during holding, rising and sinking behavior was analyzed using digital particle image velocimetry (DPIV) as in previous research (Drucker and Lauder, 1999; Wilga and Lauder, 1999; Lauder, 2000). Water in the flow tank was seeded with near-neutrally buoyant $12 \mu \mathrm{m}$ diameter silver-coated hollow glass beads (density $1.3 \mathrm{~g} \mathrm{~cm}^{-3}$, Potters Industries Inc). A $5 \mathrm{~W}$ argon-ion laser was focused into a $1-2 \mathrm{~mm}$ thick by $10 \mathrm{~cm}$ wide light sheet oriented using mirrors into a vertical configuration. Light reflected by the beads was recorded as particle movement in the water flow using a NAC two-camera high-speed video system at 250 fields $\mathrm{s}^{-1}$. The flow tank described above was used to record the swimming sharks at $1.0 \mathrm{ls}^{-1}$ (for further details, see Wilga and Lauder, 1999). Particle reflections in lateral (parasagittal) view were recorded by placing one camera perpendicular to the side of the flow tank. The position of the shark relative to the laser light sheet in posterior (frontal) view was recorded by directing a second (synchronized) camera at the surface of a mirror placed in the flow at a distance of $30-40 \mathrm{~cm}$ behind the swimming shark and positioned at $45^{\circ}$ to the side of the flow tank. Previous studies have shown that the presence of the mirror imparts no statistical effects on locomotor kinematics and does not affect the flow in the region of interest (Ferry and Lauder, 1996; Drucker and Lauder, 1999; Wilga and Lauder, 1999).

Sequences of particle images during holding, rising and sinking behavior in sharks were identified using the criteria noted above for fin kinematics. Consecutive pairs of video images ( $4 \mathrm{~ms}$ apart) of the water flow just downstream of the pectoral fin were digitized and analyzed using two-frame crosscorrelation to produce a $20 \times 20$ matrix of 400 velocity vectors, as in conventional DPIV methods (Raffel et al., 1998; Lauder, 2000). The size of the sampling window is $64 \times 64$, the sampling overlap varied between 75 and $83 \%$ because of the rectangular nature of the area of interest, and the spatial resolution of the image is $640 \times 480$. In total, 27 image pairs were analyzed for three occurrences of each behavior for each individual. Additional image pairs of the vortex after it moved further downstream were analyzed to trace the path and strength of the shed vortex when identifiable vortices attributable to the pectoral fin were visible and when such vortices were not obscured by movements of the shark body or pelvic fins.

Using the same system, Drucker and Lauder (1999) measured the accuracy of DPIV by comparing the average free-stream velocity calculated using DPIV with that calculated by tracking individual particles (PTV) and determined that the DPIV estimation of mean velocity was within $5 \%$ of the true velocity. During leopard shark locomotion, holding behavior represents the situation (with effectively free-stream flow behind the fin) for which the PTV analysis will be most similar to the DPIV analysis undertaken here. The flow during this behavior is similar to relatively uniform flow without localized flow peaks and vorticity, and is the portion of the DPIV analysis that has the most biological significance in terms of evaluating previous hypotheses of locomotion. DPIV will underestimate peak flow velocities and, hence, circulation and force for situations in which substantial local velocity peaks occur, and our estimates of circulation for rising and sinking behavior may well therefore be underestimates, while the values for holding behavior will be relatively more accurate. However, such underestimates of circulation for rising and sinking behavior will make it more difficult to detect differences among behaviors and will therefore work against our finding statistically significant differences. We did not conduct a separate error analysis of our DPIV technique to quantify the extent of the underestimate of circulation in regions of flow with large local velocity peaks.

For some analyses (such as those shown in Figs 8 and 9), we used non-rectangular subimages for the cross-correlation calculations of vectors. This was performed primarily because leopard sharks swim using considerable lateral undulation of the body, which often resulted in the pelvic fins projecting into the laser light sheet. To minimize interference by the pelvic fins (which can faintly be seen just downstream of the yellow vectors in Fig. 8), we reduced the $x$ dimension of the matrix of velocity vectors by choosing non-rectangular areas. Our choice of the shape of the interrogation area was thus determined by biological considerations. The change in shape of the crosscorrelation subimage had no significant effect on the accuracy of the velocity reconstructions. First, the free-stream velocities calculated for the non-square areas and the square areas (e.g. comparing Figs 7 and 8) were effectively identical. Because of the relatively short time interval $(4 \mathrm{~ms})$ between images and the 
relatively slow flow velocities, particles used for the crosscorrelation analyses did not move more than $20 \%$ of the box width between the two frames.

Three analytical protocols were used to quantify water flow patterns in the wake of the pectoral fin. First, fluid flow patterns were documented by estimating flow structure using the magnitude and direction of velocity vectors from plots of the $20 \times 20$ matrix of velocity vectors in the wake of the fin. Fluid structures in the wake were revealed by subtracting flowtank current from the matrix of velocity vectors.

Second, rotational motion in the flow was quantified by calculating fluid vorticity using the velocity vector matrix (Vogel and Feder, 1966; Krothapalli et al., 1997). Plots of vorticity in the present paper are shown to visualize rotational fluid motion in which the red/orange color indicates counterclockwise fluid movement, the purple/blue color indicates clockwise motion and the greenish color is used for zero vorticity (Krothapalli et al., 1997; Drucker and Lauder, 1999; Wilga and Lauder, 1999).

Third, a two-dimensional estimate of lift force generated by the pectoral fin was calculated from the velocity vector matrix. Lift forces generated by the pectoral fin were compiled from the circulation of vortices shed from the trailing edge of the fin. A useful concept in airfoil theory is Kelvin's law, which states that shed vortices are of equal and opposite strength to the hypothesized bound circulation on the fin (Dickinson, 1996). The circulation $\left(\Gamma, \mathrm{cm}^{2} \mathrm{~s}^{-1}\right)$ of vortices shed by the pectoral fins was calculated directly from the matrix of velocity vectors using a custom-designed program (Drucker and Lauder, 1999; Wilga and Lauder, 1999). The vast majority of the vortices were circular and, to conform with previous studies of fish locomotion and insect flight, we chose a circular integration path, although some vorticity plots do show a somewhat oval vortex. The circular integration area $C$ around a vortex center is defined by the user interactively against the matrix of velocity vectors. Linear interpolation of the four adjoining neighboring vectors in the matrix was used to calculate velocities at points on $C$ and by summing the tangential components around $C$ until an asymptotic value for total vortex circulation was detected by calculating increasing integration of path radii at $0.01 \mathrm{~cm}$ increments (Willert and Gharib, 1991). The location of the core center was located by a local vorticity maximum near the center of the vortex. Larger increases in path radii are used far from the maximum $(0.1 \mathrm{~cm})$ and much smaller ones near the peak $(0.01 \mathrm{~cm})$. Circulation values steadily increased from the core until the periphery of the vortex was reached, where they then started to decrease.

The lift force $F(\mathrm{~N})$ exerted by the pectoral fin on the water was calculated in two dimensions using $F=2 m t^{-1}$ (Dickinson, 1996), where 2 is included to account for the force generated by both fins, $m$ is fluid momentum (actually impulse) and $t$ is the duration of pectoral fin movement digitized from video sequences. Fluid impulse $\left(\mathrm{kg} \mathrm{m} \mathrm{s}^{-1}\right)$ was quantified using $m=\rho \Gamma A$, at a density $\rho$ of $1250\left(\mathrm{~kg} \mathrm{~m}^{-3}\right)$, and a cross-sectional area $A\left(\mathrm{~cm}^{2}\right)$ of the vortex calculated using $\pi r^{2}$, where $r$ is the digitized radius of the vortex core. In calculating forces generated by the pectoral fins, the quantity $r$ is taken as the radius of the vortex ring (Dickinson, 1996). Because some sequences captured the fin vortex after it had moved less than a vortex ring diameter downstream and since the zoomed-in video image of the wake sometimes did not show the middle of the pectoral fin, we approximated the vortex ring radius by measuring the radius of the vortex core when the shed vortex had moved approximately one radius downstream from the trailing edge of the fin. Under these conditions and given the sizes of the pectoral fins and shed vortices, the radius of the vortex core will be equal to that of the vortex ring. To provide a check on this, we measured (for those sequences for which it was possible) both the vortex core radius and the ring radius, approximated as half the distance from the vortex core to the midpoint of the pectoral fin (estimating the center of bound circulation). The mean radius of the vortex core was $1.92 \pm 0.077 \mathrm{~cm}$, while the mean radius of the ring was $1.94 \pm 0.202 \mathrm{~cm}$ (means \pm S.E.M., $N=18$ ). Given the sources of measurement error, these values are effectively identical. The difference in radii between weak and strong vortices (which we specifically included to obtain a range of biological variation during locomotion) also contributes to the standard error.

We captured the entire vortex inside the border of the image in $75 \%$ of the sequences and a partial one in the remaining $25 \%$. The reason for this inclusion of partial vortices derives from the relatively anguilliform locomotor mode of leopard sharks where other portions of the body often enter the vector field as the pectoral fin vortex drifts downstream. No vortex was used that was cut off at the center or less than the center. Most importantly, we captured the entire region just behind the fin and inside the border of the image in all the position-holding sequences for which circulation values are expected to be (and were) the lowest. The integration routine does not supply data for missing vectors; it calculates circulation only for visible vectors within the designated radius. We realize that this provides an underestimate of the lift force for those sequences with missing vectors but, since the circulation values for sequences with missing vectors fall within the range of the values for sequences for which no vectors were missing, they were not considered outliers. In other words, the range of biological variation for a given behavior is greater than the variation introduced by missing a portion of the vortex in $25 \%$ of the sequences. We analyzed rising and sinking behaviors that may be described as weak to strong deliberately to encompass the range of biological locomotor variation, and the associated vortices and calculated values for lift varied accordingly.

The vertical fluid impulse of the downwash behind the fin was calculated using fluid momentum data obtained from the laser light sheet, which provided a two-dimensional reconstruction of vortex geometry. These calculations permit comparative analysis of vortex properties and wake momentum among the three behaviors, but do not constitute total lift force on the fin, for which three-dimensional data and upstream flow profiles are required (Prandtl and 
Tietjens, 1934; Drucker and Lauder, 1999; Wilga and Lauder, 1999). On the basis of our two-dimensional analysis, we recognize that our reported values will not provide an accurate estimate of the absolute magnitude of lift generated by the pectoral fin and that the lift values for rising and sinking behavior may be underestimates. The key issue for us in the present paper is the comparative values obtained from the same fish during three different locomotor behaviors. The sources of error in estimating lift force work against finding significant differences among behaviors; therefore, if such differences are found, we feel confident of their biological significance.

Fin wake vortices were typically observed following movement of the posterior region of the pectoral fin (triangle $\beta$ ). The velocity of triangle $\beta$ movement was calculated for sequences in which the wake vortex force was estimated to compare the velocity of fin motion with the magnitude of estimated lift forces and among the three behaviors. We measured the vortex an average of $45 \mathrm{~ms}$ after it left the fin trailing edge. According to the viscous length scale (Acheson, 1990), vorticity diffuses a distance of $0.022 \mathrm{~cm}$ in $45 \mathrm{~ms}$, and thus we believe that viscous dissipation is not significantly affecting our circulation estimates.

\section{Statistical tests}

Linear regression analyses were calculated using the following dependent and independent variables and adjusted $r^{2}$ values: whole body angle and flow speed; anterior base of second dorsal fin position and proportion of tailbeat; threedimensional pectoral fin angle and body angle; lift force and fin flip velocity. For the whole body and head and pectoral fin variables versus behavior analyses, a mixed-model two-way analysis of variance (ANOVA) using Type III sums of squares was performed on the behavior data (Hicks, 1982; SAS, 1998). Behavior was treated as a fixed main effect and individuals as a random main effect. Behavior was tested over the behavior $x$ individual interaction term. If a difference was detected by ANOVA, then a Student-Newman-Keuls multiple-comparisons test was performed on the data. The data were tested for homogeneous variances using the Levene median test $(P<0.05)$ and normal distribution using the Kolmogorov-Smirnov test $(P<0.05)$. Statistical tests were performed using SAS v. 6.12 or SigmaStat v. 2.01 statistical software or were calculated using Zar (1996).

\section{Results \\ Body and pectoral fin kinematics}

Swimming sharks held position in the flow tank using continuous undulations of the body and tail at a positive body tilt relative to the flow that decreases with increased speed (Fig. 2). Body tilt averaged $11^{\circ} \pm 1.66$ S.E.M., $N=12$ at $0.5 l \mathrm{~s}^{-1}$ and decreased to $0.6^{\circ} \pm 0.90$ s.E.M., $N=12$ by $2.0 l \mathrm{~s}^{-1}$. The linear regression was significant with an $r^{2}$ of $0.43 \quad(P<0.001)$. Significantly higher body angles were adopted by swimming sharks at decreasing flow speeds (ANOVA, $P=0.005$ ).
Sharks swam horizontally with no pitching or vertical or lateral drifting during holding sequences, and smoothly rose or sank (instead of rolling or yawing) during the rising and sinking sequences as confirmed by plots of $y$-coordinate values of five points digitized along the body (points $2-6$ on Fig. 1)
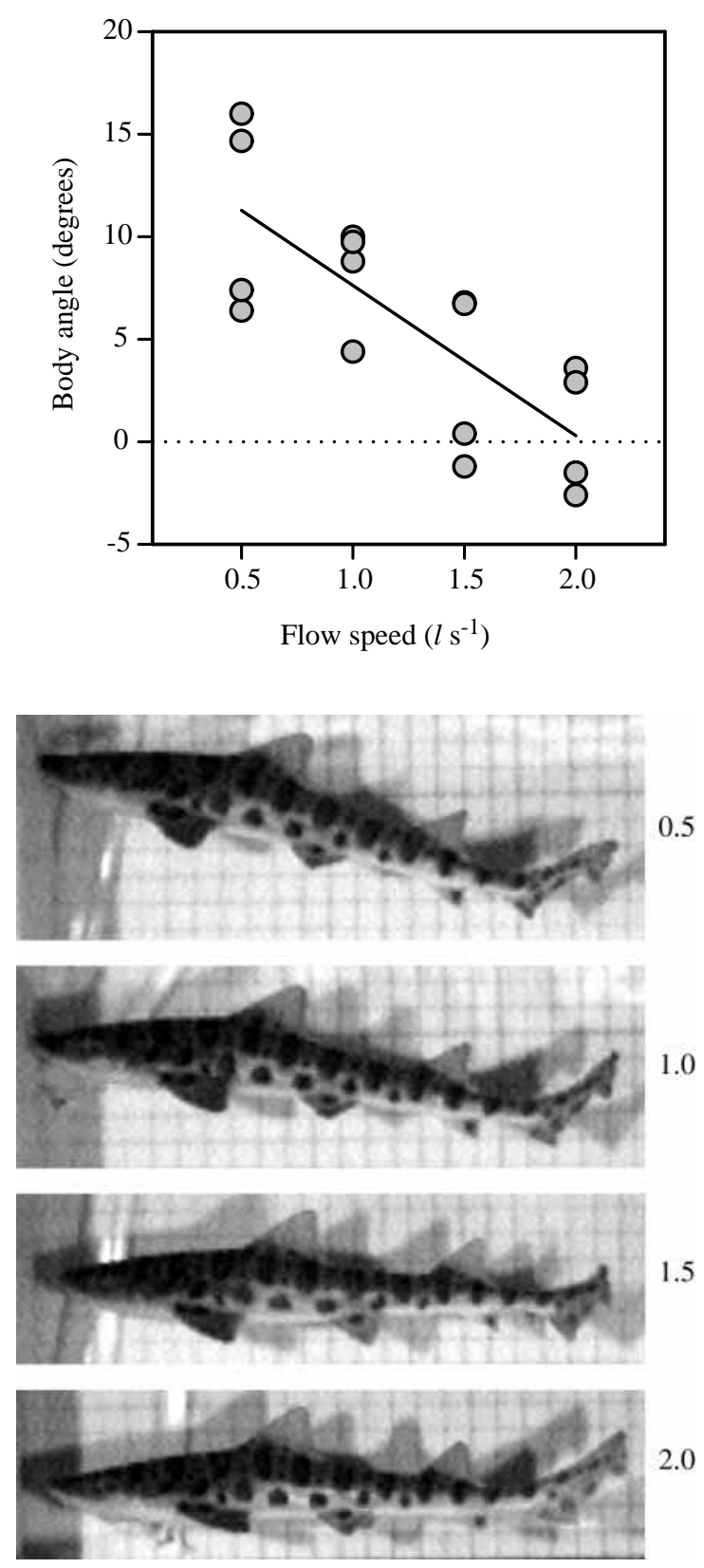

Fig. 2. Plot of body angle versus flow speed to show the decreasing angle of the body with increasing speed. Each symbol represents the mean of five body angle measurements (equally spaced in time) for five tailbeats for four individuals. Images below show body position at the corresponding flow speeds in $l \mathrm{~s}^{-1}$, where $l$ is total body length (flow direction is left to right). At all speeds, sharks are holding both horizontal and vertical position in the flow, and not rising or sinking in the water column. Body angle was calculated using the line connecting points 7 and 9 (Fig. 1) and the horizontal (parallel to the flow). A linear regression $\left(y=15.1-7.4 x\right.$, adjusted $\left.r^{2}=0.43 ; P<0.001\right)$ was significant and gives the best fit to the data. 
versus time. Incremental vertical changes during the tailbeat sequence from an initial point position of 0 were tested for the $y$ position of points 2-6 during rising, holding and sinking behaviors at $1.0 l \mathrm{~s}^{-1}$. Least-squares linear regression revealed that the slopes are significantly different for each of the three behaviors: rising $y=-0.059+3.71 x$; holding $y=-0.043+0.097 x$; sinking $y=0.29-4.39 x \quad(P<0.001)$, where $x$ is time. Furthermore, the slope is not significantly different from zero for holding behavior $(P=0.36)$. Rising and sinking sequences were initiated by pitching movements caused by changes in pectoral fin angle as described below.

The angle of the body relative to the flow varied significantly while changing position in the water column at $1.0 \mathrm{l} \mathrm{s}^{-1}$ (ANOVA, $P=0.0001$ ) (Fig. 3; Table 1). A positive body tilt averaging $22.0^{\circ}$ was assumed during rising, whereas a negative body tilt averaging $-11.1^{\circ}$ was adopted during sinking, and a positive body tilt averaging $8.3^{\circ}$ was maintained while holding position. Overall mean tailbeat duration was $545 \pm 54 \mathrm{~ms}$, within which sharks actively increased or decreased vertical position by an average of $8.1 \pm 1.9 \mathrm{~cm} \mathrm{~s}^{-1}$ during rising and $12.0 \pm 2.7 \mathrm{~cm} \mathrm{~s}^{-1}$ (means \pm S.E.M., $N=20$ ) during sinking. The conformation of the pectoral fin surface also changed significantly during the initiation of sinking and rising from an initial holding position at $1.0 \mathrm{ls}^{-1}$ as revealed by three-dimensional analyses of pectoral fin angles (ANOVA, $P=0.0001$; linear regression, internal pectoral fin angle $=190.824+0.414 x$, where $x$ is body angle, $r^{2}=0.39, P<0.001$; Fig. 4). Conversely, pectoral fin

Fig. 3. Plot of body angle versus behavior during locomotion at $1.0 l \mathrm{~s}^{-1}$, where $l$ is total body length. Circles indicate holding behavior, triangles show rising behavior and squares reflect sinking behavior. Body angle was calculated using the line connecting points 7 and 9 (Fig. 1) and the horizontal (parallel to the flow). Each point represents the mean of five sequences for each of four individuals. Images below show a sample body position during rising, holding and sinking behaviors. Body angle is significantly different among the three behaviors (ANOVA, $P=0.0001$ ). conformation does not change throughout the tail-beat cycle while holding. During holding behavior, the pectoral fin is held at a mean internal angle of $191^{\circ}$ (Table 1) between
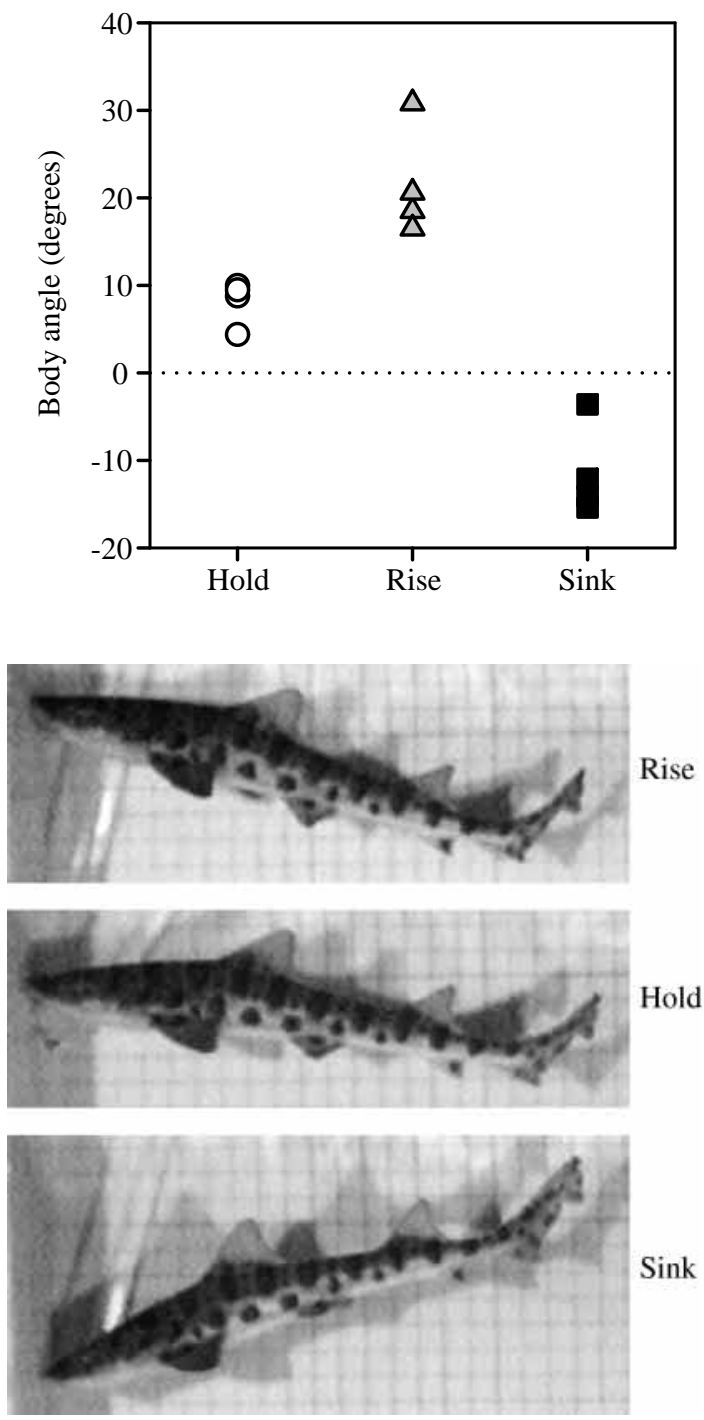

Table 1. Summary means of kinematic and DPIV variables while holding and changing position at $1.01 \mathrm{~s}^{-1}$ in two fish species with heterocercal tails

\begin{tabular}{|c|c|c|c|c|c|c|}
\hline \multirow[b]{2}{*}{ Variable } & \multicolumn{3}{|c|}{ Triakis semifasciata } & \multicolumn{3}{|c|}{ Acipenser transmontanus } \\
\hline & Hold & Rise & Sink & Hold & Rise & Sink \\
\hline Body tilt angle relative to flow (degrees) & $8 \pm 0.80$ & $22 \pm 1.75$ & $-11 \pm 1.93$ & 8 & 19 & -10 \\
\hline Pectoral fin chord angle to flow (degrees) & $-5 \pm 1.07$ & $14 \pm 1.61$ & $-22 \pm 1.57$ & -11 & 12 & -29 \\
\hline Dihedral ( $\alpha$ plane) (degrees) & $-23 \pm 2.41$ & $-35 \pm 1.91$ & $-5 \pm 3.62$ & -33 & -34 & -26 \\
\hline
\end{tabular}

Data for Acipenser transmontanus are from Wilga and Lauder (1999).

$l$, total body length; for definitions of $\alpha$ and $\beta$, see Fig. 1 .

Values are means \pm S.E.M. of 300 kinematic sequences ( 5 fields for 5 tailbeats for 3 behaviors for 4 individuals, $N=4$ ) and 27 DPIV sequences (3 fields for 3 behaviors for 3 individuals, $N=3$ ). 

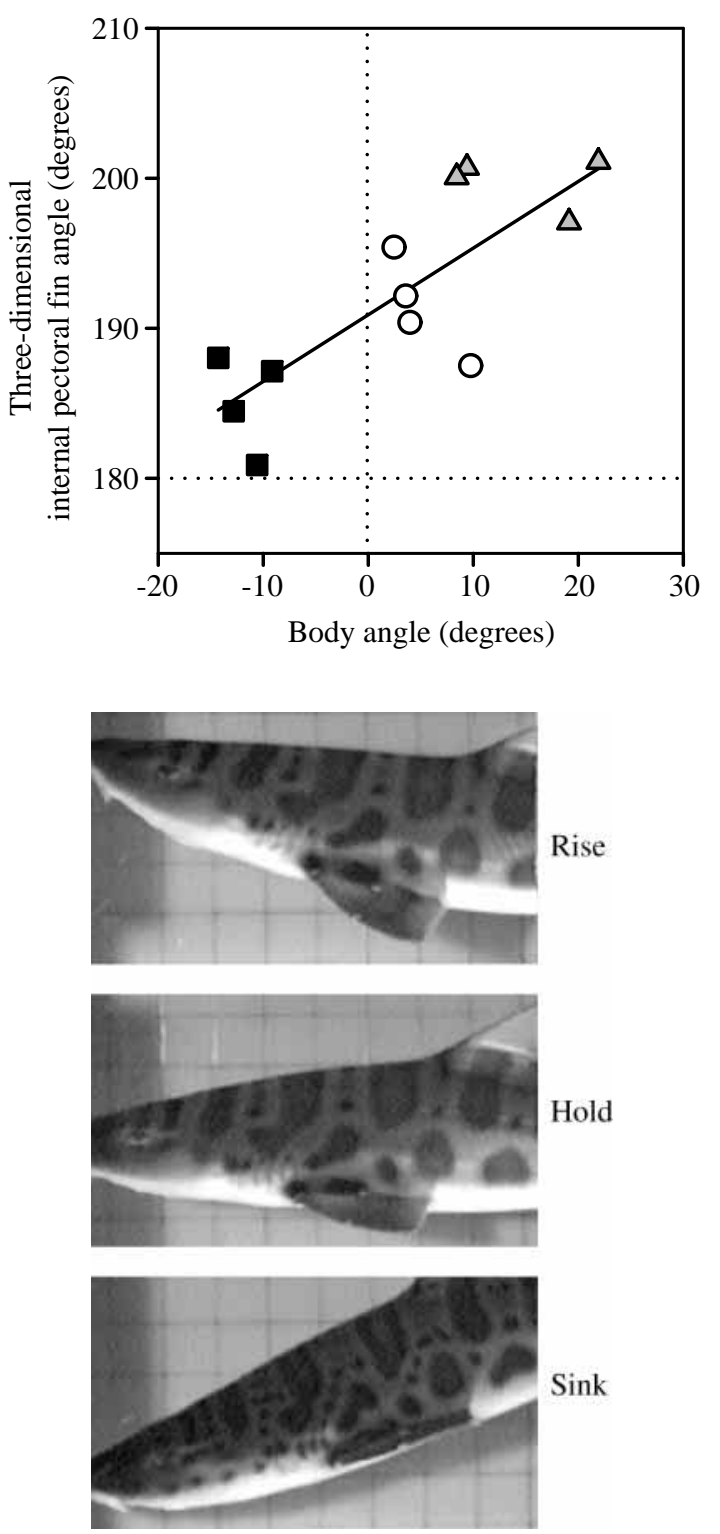

Fig. 4. Graph of three-dimensional internal pectoral fin angle versus body angle for each of the three behaviors during locomotion at $1.0 l \mathrm{~s}^{-1}$, where $l$ is total body length. Symbols are as in Fig. 3. Body angle was calculated using the line connecting points 12 and 13 (see Fig. 1) and the horizontal (parallel to the flow). Each point represents the mean of five sequences for each of four individuals. Images below show sample head and pectoral fin positions during rising, holding and sinking behaviors. Pectoral fin angles equal to $180^{\circ}$ indicate that the two fin triangles (see Fig. 1C) are coplanar; angles less than $180^{\circ}$ show that the fin surface is concave dorsally; angles greater than $180^{\circ}$ indicate that the fin surface is concave ventrally. The three-dimensional internal pectoral fin angle is significantly different among the three behaviors (ANOVA, $P=0.0001$ ). The leastsquares regression line is significant (slope $0.41, P<0.001$; adjusted $\left.r^{2}=0.39\right)$.

planes $\alpha$ and $\beta$, indicating that the fin is retained in a slightly concave downward orientation. Note that if the internal angle were $180^{\circ}, \alpha$ and $\beta$ would be coplanar and the fin would be
Table 2. Summary statistics of three-dimensional planar angles in the pectoral fin in Triakis semifasciata while holding position at $1.01 \mathrm{~s}^{-1}$

\begin{tabular}{lcrrrrr}
\hline Plane & $\mathrm{T}$ & $\begin{array}{c}\text { Holding } \\
\text { (degrees) }\end{array}$ & $\begin{array}{c}\text { Rising } \\
\text { (degrees) }\end{array}$ & $\begin{array}{c}\text { Sinking } \\
\text { (degrees) }\end{array}$ & $P$ & $\mathrm{SNK}$ \\
\hline$X Z$ & $\alpha$ & $149 \pm 4.2$ & $217 \pm 1.8$ & $146 \pm 1.5$ & $0.0001^{*}$ & $\mathrm{R}>\mathrm{H}>\mathrm{S}$ \\
$X Z$ & $\beta$ & $207 \pm 8.4$ & $217 \pm 1.6$ & $151 \pm 2.0$ & 0.0033 & $\mathrm{R}>\mathrm{H}>\mathrm{S}$ \\
$Y Z$ & $\alpha$ & $87 \pm 0.7$ & $105 \pm 1.8$ & $67 \pm 1.2$ & $0.0001^{*}$ & $\mathrm{R}>\mathrm{H}>\mathrm{S}$ \\
$Y Z$ & $\beta$ & $96 \pm 1.8$ & $115 \pm 1.6$ & $74 \pm 2.0$ & $0.0001^{*}$ & $\mathrm{R}>\mathrm{H}>\mathrm{S}$ \\
$X Y$ & $\alpha$ & $113 \pm 2.4$ & $125 \pm 1.9$ & $95 \pm 3.6$ & $0.0008^{*}$ & $\mathrm{R}>\mathrm{H}>\mathrm{S}$ \\
$X Y$ & $\beta$ & $114 \pm 7.7$ & $119 \pm 1.7$ & $90 \pm 3.4$ & 0.0279 &
\end{tabular}

Values are means \pm S.E.M. of 300 kinematic sequences ( 5 fields for 5 tailbeats for 3 behaviors for 4 individuals, $N=4$ ).

*Significant at the Bonferroni-corrected $P$-value of 0.008. SNK, results of Student-Newman-Keuls test; $\mathrm{H}$, hold; R, rise; S, sink.

In the $X Z$ plane, $0^{\circ}$ is anterior and $180^{\circ}$ is posterior; in the $Y Z$ and $X Y$ planes, $0^{\circ}$ is dorsal and $180^{\circ}$ is ventral.

Note that the orientation of the $\beta$ triangle to the perpendicular of the $Y Z$ plane represents the plane of the posterior portion of the pectoral fin relative to the flow (see Fig. 1 for a definition of the $\beta$ plane).

held like a rigid flat plate. The internal angle increases to an average of $200^{\circ}$ during rising (Table 1), indicating that the fin is held in an even greater concave-down position, while the fin is less concave with a mean internal angle of $185^{\circ}$ during sinking. In addition, the leading edge of the pectoral fin is retracted to $170 \pm 3.2^{\circ}$ during rising and protracted to $152 \pm 4.5^{\circ}$ during sinking (ANOVA, $P<0.001$ ) from the mean angle during holding of $158 \pm 2.2^{\circ}$ (the angle between lateral side of head and anterior margin of fin, angle formed by points $15 \mathrm{v}, 14 \mathrm{v}$ and 18 ) (means \pm S.E.M., $N=20$ ). The repositioning of the pectoral fin to redirect water flow to reorient the body for rising and sinking behaviors is reflected in the change in the internal angle. Changes in internal pectoral fin angle precede the change in body angle associated with rising or sinking and function to initiate the pitching moment that reorients the body angle from the holding position to the more extreme body angles associated with rising and sinking behavior (Fig. 4).

The movement of the pectoral fins during locomotion in sharks is complicated and characterized best by illustrating the angle of the pectoral fin planes with respect to the three external reference planes (Table 2; Fig. 5). Pectoral fin plane $\alpha$ was held outwards from the body at a mean angle of $67^{\circ}$ from the parasagittal $(X Y)$ plane while holding position, as measured ventrally (or $113^{\circ}$ as measured dorsally, see Table 2). The leading fin edge is actually ventral to the trailing edge with the fin chord inclined at an angle of $-5^{\circ}$ to the flow (Fig. 6A; Table 1). The angle of attack of the pectoral fin is thus negative during holding behavior, and the lateral tip of the fin is significantly ventral to the base and posterior margin of the fin.

During rising, the pectoral fin tilts so that the leading edge is dorsal to the trailing edge, and the fin chord is at a positive 

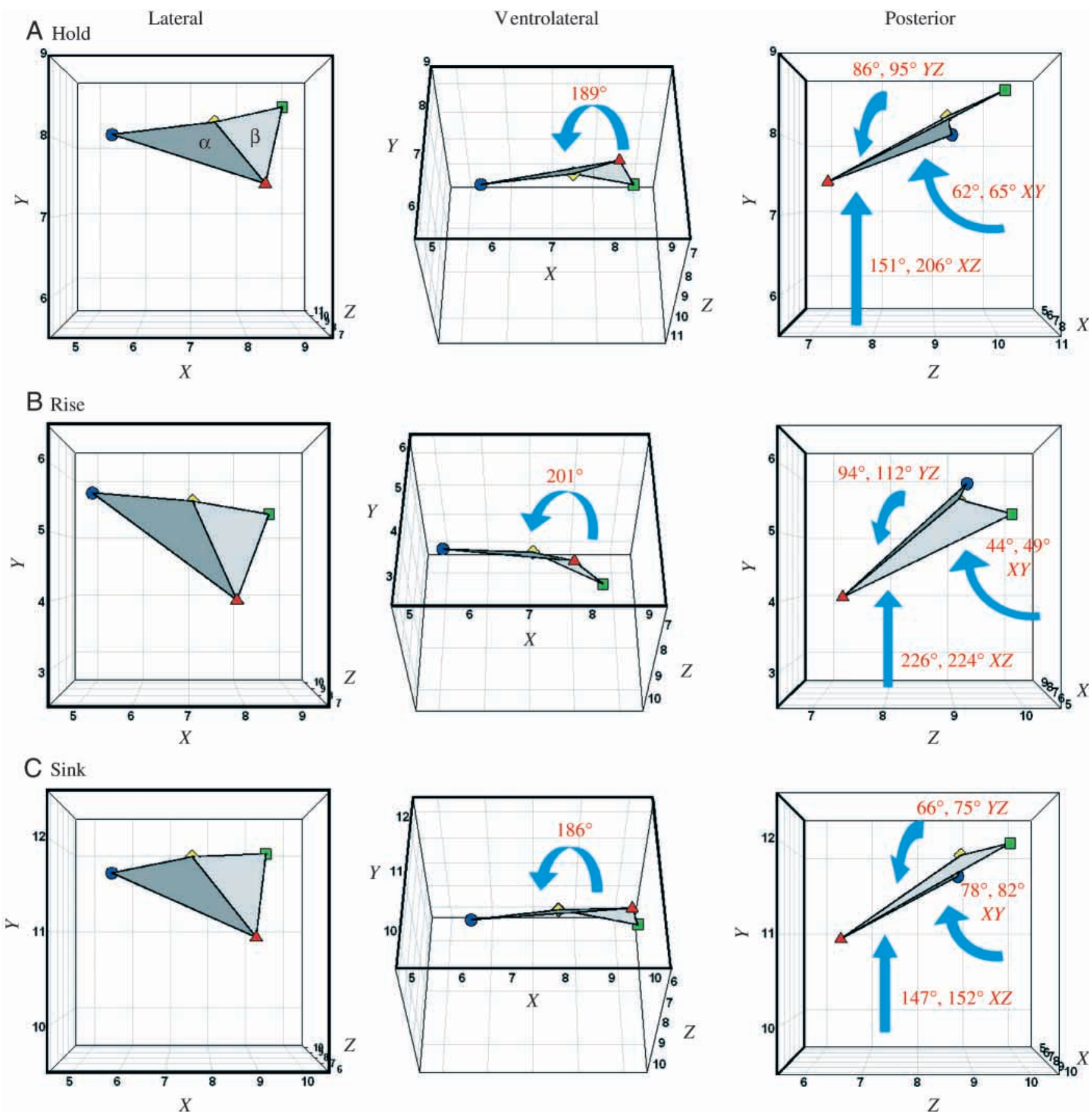

Fig. 5. Orientation of the two pectoral fin planes $(\alpha$ and $\beta)$ in three-dimensional space during holding, rising and sinking behaviors. The three columns show lateral, ventrolateral and posterior views of the fin triangles respectively. Points defining the fin triangles correspond to the following digitized locations in Fig. 1: blue circle, point 14; red triangle, point 15; yellow diamond, point 17; green square, point 16. The homologous $X Y$ plane (the earth plane of reference) is outlined in bold in each panel to assist in identifying the rotational view. The internal fin angle between planes $\alpha$ and $\beta$ is given in the ventrolateral view column, and the angles of each fin triangle are given with respect to the three external reference planes in the posterior view column. The first and second numbers indicate the external three-dimensional angles of the $\alpha$ and $\beta$ triangles respectively; note that in the posterior view column the angles are given as acute to the $X Y$ plane and thus are the complement to the angles reported in Table 1. Values reflect orientation in these three specific sequences, rather than the overall means reported elsewhere.

$14^{\circ}$ to the flow (Figs $5 \mathrm{~B}, 6 \mathrm{~B}$; Table 1 ). The orientation of the pectoral fin during sinking changes significantly from its conformation while holding with respect to all three reference planes (Table 2). The lateral edge of the pectoral fin is more dorsally located, the posterior plane of the fin slants upwards relative to the base, and the fin chord is oriented at an angle of attack of $-22^{\circ}$ to the flow relative to the holding position (Figs 5C, 6C; Table 1). 

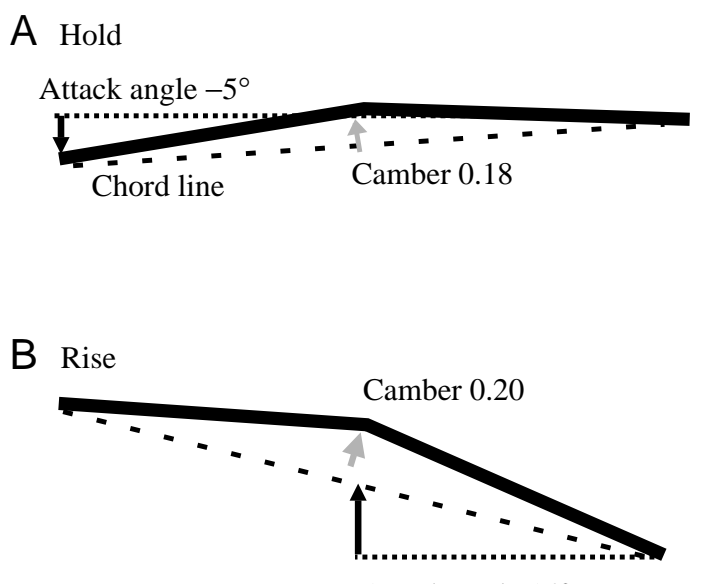

Attack angle $14^{\circ}$

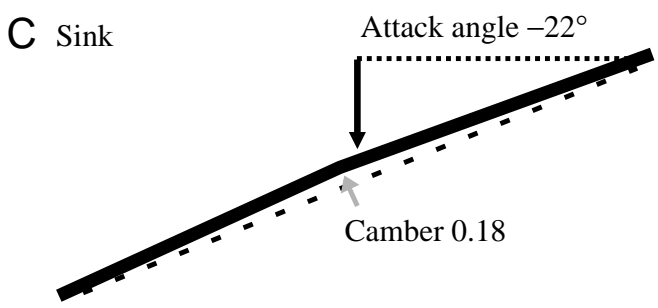

Fig. 6. Schematic diagram of pectoral fin chord, camber and orientation during holding, rising and sinking behaviors. The pectoral fin is represented as two planes $(\alpha+\beta$; see Fig. 1 for definitions) seen laterally, and camber is calculated as described by Kundu (1990). Note that, during steady horizontal swimming (holding behavior), the pectoral fin has a negative angle of attack and is inclined downwards with respect to the flow, which is parallel to the horizontal dotted line. The angle of attack is given between the chord line (dashed line) and the flow (dotted line).

\section{Digital particle image velocimetry (DPIV)}

Digital particle image velocimetry was used to visualize the pattern of water flow in the wake of the pectoral fin during the three behaviors. Video images were superimposed with the corresponding velocity vector field, and the calculated vorticity matrix is shown for the three behaviors in Figs 7-9. The body of the shark was often faintly visible through the light sheet, while the left pectoral fin was clearly visible as it extended through (or just anterior to) the light sheet.

The DPIV analyses reveal downstream-oriented vectors and largely solid green vorticity fields, indicating that essentially no vorticity was detected in the wake of the pectoral fins of leopard sharks during holding (Fig. 7). In contrast, as leopard sharks rose in the water column, a counterclockwise vortex (Fig. 8: the red/yellow center of vorticity on right) was generated by the downward flipping of the posterior portion of the pectoral fin and shed from the trailing edge of the fin to initiate the upward pitching of the body. Similarly, a clockwise vortex (negative vorticity) was produced as the posterior portion of the pectoral fin flipped upwards to initiate a sinking event in leopard sharks (Fig. 9). The blue region of rotating fluid in the vorticity plot indicates the position of a clockwise vortex.

Mean values for relative downstream vertical fluid impulse and velocity of pectoral fin movement for the three behaviors were calculated from the velocity vector matrix and from video images (Table 1). Mean force values quantified while holding position were not significantly different from zero (mean force $0.0009 \mathrm{~N} ; t$-test $P=0.493)$. The counterclockwise vorticity observed in the wake of the pectoral fin during rising corresponds to a significant positive force that acts to pitch the body upwards (mean force $0.062 \mathrm{~N}$; $t$-test $P<0.001$ ). Similarly, the clockwise vorticity observed in the wake of the pectoral fin during sinking corresponds to a negative force that acts to pitch the body downwards and is significantly different from zero (mean force $-0.0925 \mathrm{~N} ; t$-test $P<0.001$ ).

Relative two-dimensional forces produced by the flipping of the pectoral fin are significantly different among the three behaviors (ANOVA, $P=0.01$ ). The least-squares linear regression of fin flip velocity versus downstream vertical fluid impulse was also significant $\left(r^{2}=0.22, P=0.007\right)$ (Fig. 10). Holding events, in which the fin is held constant and does not flip up or down, cluster around zero force and zero fin flip velocity. Rising events are generated by downward fin flips and have positive values (negative velocities) and are located in the upper left quadrant of Fig. 10. In contrast, sinking events are associated with an upward fin flip and have negative lift forces (positive velocities by our convention) and are located in the lower right quadrant of Fig. 10.

\section{Discussion \\ Body position effects}

Steady horizontal swimming in $T$. semifasciata is characterized by continuous undulations of the body and caudal fin with the body tilted at a positive angle to the flow. It has been suggested that negatively buoyant fishes may adopt a positive body tilt during steady horizontal swimming as a behavioral mechanism to increase total lift (He and Wardle, 1986). The positive tilt of the body is used as a hydrofoil to increase the total body area generating lift, particularly at low swimming speeds (less than $1.0 l \mathrm{~s}^{-1}$ ) when lift from the pectoral fins is presumably insufficient. In addition, mean body tilt during steady swimming in $T$. semifasciata steadily decreased from $11^{\circ}$ at $0.5 l \mathrm{~s}^{-1}$ to $0.6^{\circ}$ at $2.0 l \mathrm{~s}^{-1}$. Leopard sharks thus appear actively to alter body tilt to modulate the amount of lift generated by the body profile as needed. Illustrations in the literature of body posture during steady swimming in sharks typically show fish swimming with the ventral body surface oriented horizontally, parallel to the flow (Breder, 1926; Harris, 1936; Aleev, 1969; Thomson and Simanek, 1977). However, in the flow tank, leopard sharks swim with a positive body angle of attack at speeds less than $2.0 l \mathrm{~s}^{-1}$. This positive body angle posture is crucial to the total force balance during steady swimming as well as in maneuvering, as discussed below. Dorsal views of swimming sharks will not reveal the tilted posture, and changes in body 
orientation with speed may not be detected with such camera views.

Tailbeat frequency $(f)$ increased linearly, as in most fish, from $1.2 \mathrm{~Hz}$ at $0.5 l \mathrm{~s}^{-1}$, to $2.0 \mathrm{~Hz}$ at $1.0 l \mathrm{~s}^{-1}$, to $3.0 \mathrm{~Hz}$ at $1.5 \mathrm{l} \mathrm{s}^{-1}$ and to $3.5 \mathrm{~Hz}$ at $2.0 l \mathrm{~s}^{-1}$ (Johnson, 1970; Webb and Keyes, 1982). This is similar to the tailbeat frequency of $1.1 \mathrm{~Hz}$ reported for larger $T$. semifasciata ( $98 \mathrm{~cm}$ total length) swimming at $0.58 \mathrm{ls}^{-1}$ in large still-water aquaria (Webb and Keyes, 1982).

The tilt angle of the body was also altered when $T$. semifasciata changed position in the water column at $1.0 \mathrm{l} \mathrm{s}^{-1}$. Mean body tilt was increased to $22^{\circ}$ during rising and decreased to $-11^{\circ}$ when sinking. These alterations in body tilt facilitate rising and sinking by altering the total force balance on swimming sharks to allow changes in vertical position in the water column.

\section{Role of the pectoral fins during steady swimming}

In contrast to predictions of previous hypotheses of pectoral fin function in sharks, we were unable to detect any evidence of lift in the wake structure behind the pectoral fins of $T$. semifasciata during steady horizontal swimming at $1.0 l \mathrm{~s}^{-1}$ with our methods. The planar surface of the pectoral fin is held concave downwards relative to the flow during steady horizontal swimming. Thus, the pectoral fin has camber with a mean dorsal obtuse angle of $191^{\circ}$ between the two planes of the fin. Furthermore, the angle of attack between the direction of flow and the chord line of the pectoral fin is on average $-5^{\circ}$ during steady horizontal swimming (Fig. 6A). Therefore, the pectoral fins of leopard sharks are held in such a way to generate negligible lift or even potentially slight negative lift, in contrast to previous assumptions that the pectoral fins of sharks during steady horizontal swimming generate positive lift forces.

The results of the DPIV analyses of the pectoral fin wake further corroborate the conclusion from the kinematic data that the pectoral fins of leopard sharks generate negligible lift during steady horizontal swimming. There was virtually no vorticity and downwash detected in the wake of the pectoral fins of leopard sharks during steady horizontal swimming, suggesting that little or no lift is being produced by the fins (Table 1). According to Kelvin's law, vortices shed from the pectoral fin must be equivalent in magnitude but opposite in direction to the theoretical bound circulation around the fin (Kundu, 1990; Dickinson, 1996). Therefore, the circulation of the shed vortex can be used to estimate the force on the fin. We calculated lift in only one plane so that relative comparisons among the three behaviors could be made (our calculations do not reveal the total force on the fin). Mean downstream vertical fluid impulse calculated in the wake of the pectoral fins during steady horizontal swimming was $0.0009 \mathrm{~N}$, which is not significantly different from zero ( $t$-test, $P=0.493$ ) (Table 1). This indicates that leopard sharks are holding their pectoral fins in such a way that the flow speed and pressure are equivalent on the dorsal and ventral surfaces of the fin. Furthermore, if the pectoral fins were generating substantial lift to counteract moments generated by the heterocercal tail, there must be a downwash behind the wing to satisfy Kelvin's law. The lack of an observable downwash indicates clearly that, during holding behavior, the pectoral fins generate negligible lift.

The posture of the body and pectoral fins relative to the flow during steady horizontal swimming in leopard sharks contrasts with the position of the fuselage and wings of a cruising airplane. According to our conventions, the direction of flow is perpendicular to the $Y Z$ plane, and hence an acute $Y Z$ angle would indicate a negative angle of attack relative to the flow, while an obtuse angle would indicate a positive angle of attack (Fig. 5). The anterior and posterior planes of the pectoral fin in leopard sharks during holding make acute and obtuse angles, respectively, with the $Y Z$ plane (Table 2), and the anterior plane is held at a negative attack angle and the posterior plane at a slight positive attack angle to the flow. When both planes are considered together, the angle of the chord line is $-5^{\circ}$. Conversely, the wings of most cruising airplanes make an obtuse angle to the $Y Z$ plane and hence possess a positive attack angle to the direction of oncoming air, which generates positive lift.

Our findings that the pectoral fins of sharks do not generate a flow downwash during steady forward swimming stand in contrast to previous suggestions (Daniel, 1922; Harris, 1936; Aleev, 1969). Daniel (1922) described experiments in which a rubber band was placed over the pectoral fins of a juvenile shark, which resulted in downward swimming. From this, Daniel concluded that the pectoral fins function to allow horizontal swimming by directing water downwards. Pectoral fin amputation experiments on dusky smoothhounds Mustelus canis and spiny dogfish Squalus acanthias also resulted in downward swimming of the fish (Harris, 1938; Aleev, 1969). Horizontal swimming was resumed only when the fish elevated its head and ultimately ended with the fish swimming at the surface with its head out of water and its body at $45^{\circ}$ to the flow (Harris, 1938). Although the results of such radical experiments are difficult to evaluate because of various possible reactions to surgery and compensatory action by the shark, it is likely that the lack of pectoral fins prevented the sharks from initiating changes in pitch using the mechanisms discussed below and, hence, limited their ability to achieve a horizontal position and adjust to perturbances in oncoming flow.

Lift forces measured on the pectoral fins and body of a plaster model of Mustelus canis in a wind tunnel also suggested that the pectoral fins generated upward lift while the body generated no lift (Harris, 1936). However, the pectoral fins were modeled as rigid flat plates that were tilted upwards at $8^{\circ}$ to the flow and perpendicular to the longitudinal axis of the body, which was oriented at $0^{\circ}$ to the flow. Pectoral fins placed in this orientation must generate a downwash while the body would not. Although it is possible that Mustelus canis locomotes with the body and pectoral fins in this position, the results of the present study on the closely related Triakis semifasciata, a shark with very similar fin and body morphology, show a radically different orientation of the body and pectoral fins in vivo: that of a cambered, downward-tilted pectoral fin in a negative dihedral orientation (negative angle 

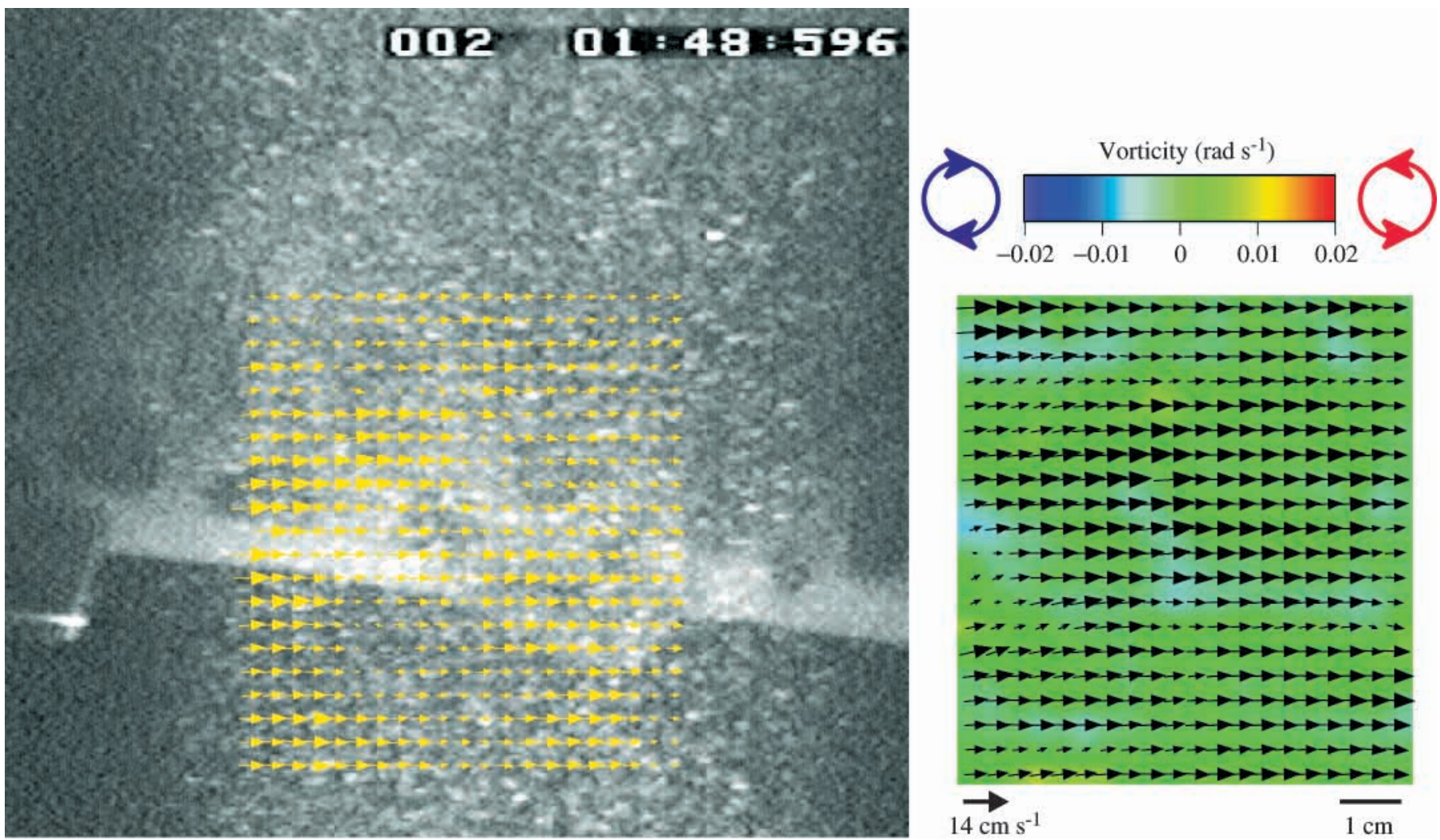

Fig. 7
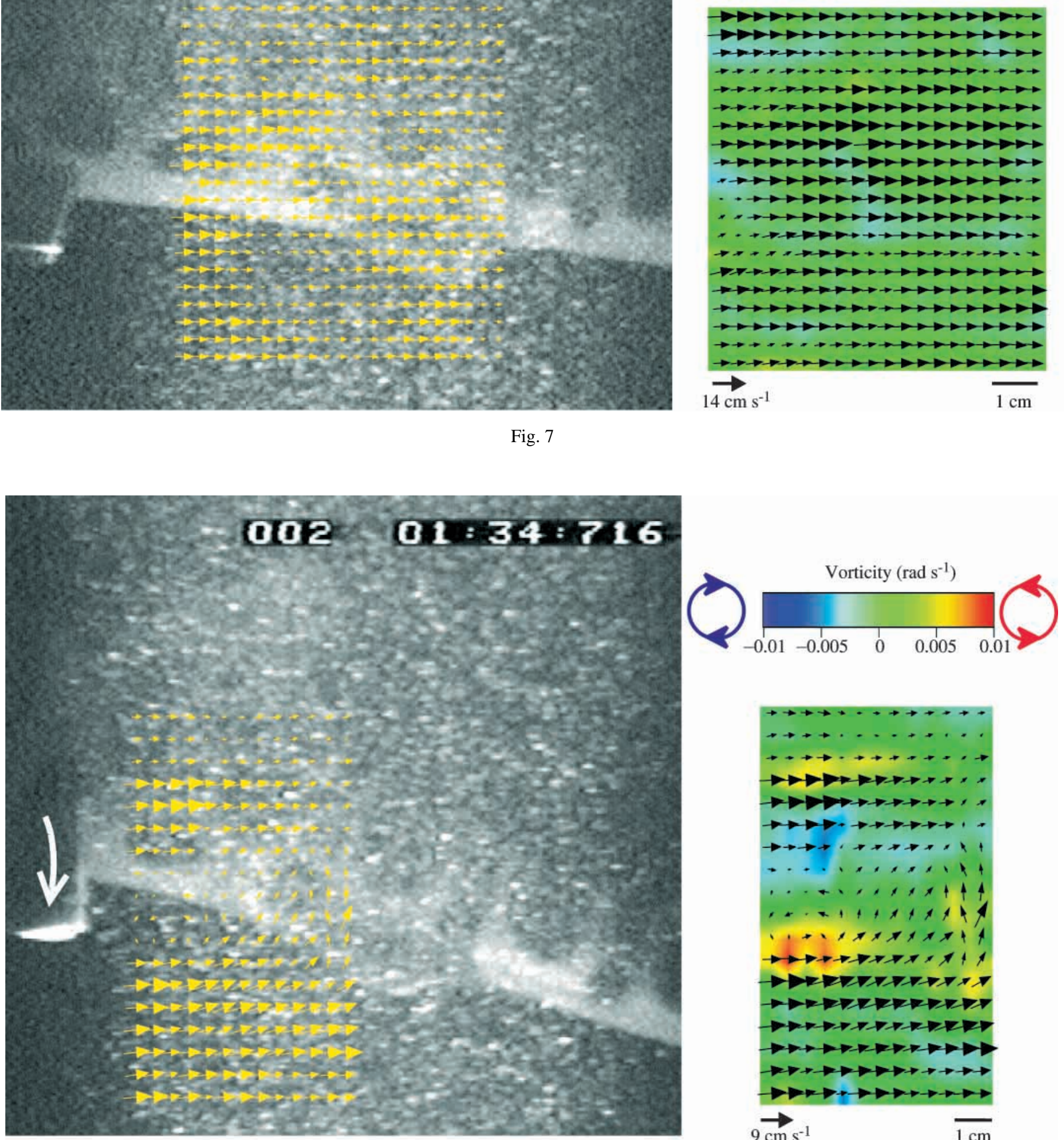

Fig. 8

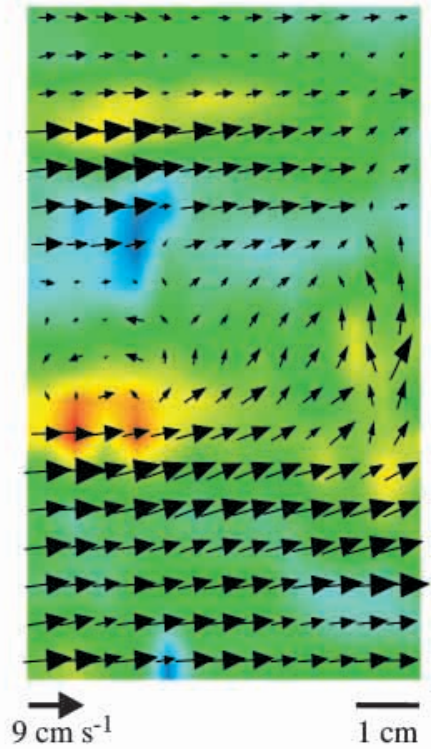


Fig. 7. Digital particle image velocimetry (DPIV) data from shark pectoral fins during holding behavior. The video image (on the left) is a single image of a shark with the left pectoral fin located just anterior to the laser light sheet. Note that the ventral body margin is faintly visible through the light sheet. The $20 \times 20$ matrix of yellow vectors downstream of the pectoral fin represents the results of DPIV calculations based on particle images visible as the speckled pattern in the light sheet. The plot on the right shows fluid vorticity with superimposed velocity vectors (scaled to the same size as the vector matrix in the video image). Note that the fin is held in a horizontal position, and that the vorticity plot (scaled to the same values as Fig. 9) shows effectively no fluid rotation. Hence, the pectoral fins in this position do not generate lift forces. Note that

to the horizontal plane $X Y$ ) with the body tilted at a positive angle to the flow.

\section{Role of the pectoral fins during vertical maneuvering}

Leopard sharks actively adjust the angle of their pectoral fins to maneuver vertically in the water column. Angular adjustments of the pectoral fins are used to generate negative and positive lift forces, which then initiate changes in the angle of the body relative to the flow. Rising in the water column is initiated when the posterior plane $(\beta)$ of the fin is flipped downwards to produce a mean obtuse fin internal angle of $200^{\circ}$ the green color indicates no fluid rotation, the blue color reflects clockwise fluid rotation and the red/yellow color indicates counterclockwise fluid rotation. To assist in visualizing the flow pattern, a mean horizontal flow of $U=33 \mathrm{~cm} \mathrm{~s}^{-1}$ was subtracted from each vector.

Fig. 8. Digital particle image velocimetry (DPIV) data from leopard shark pectoral fins at the initiation of rising behavior. Conventions as in Fig. 7. Note that the fin has flipped ventrally (curved white arrow) to initiate the rising event, and that a counterclockwise vortex (orange/red vorticity on the right) has been shed from the fin. To assist in visualizing the flow pattern, a mean horizontal flow of $U=35 \mathrm{~cm} \mathrm{~s}^{-1}$ was subtracted from each vector.

(Table 1), while the leading edge of the fin is rotated upwards relative to the flow. This downward flipping of the posterior portion of the fin increases the chord angle of attack to $+14^{\circ}$ (Fig. 6B). As the posterior plane of the pectoral fin is flipped down, a counterclockwise vortex is produced and shed from the trailing edge of the fin (Fig. 8). This vortex is readily visible in the wake and visualized using DPIV as it rolls off the fin and is carried downstream.

The opposite wake flow pattern occurs when leopard sharks initiate a sinking maneuver in the water column. In this case, the posterior plane of the pectoral fin is flipped upwards
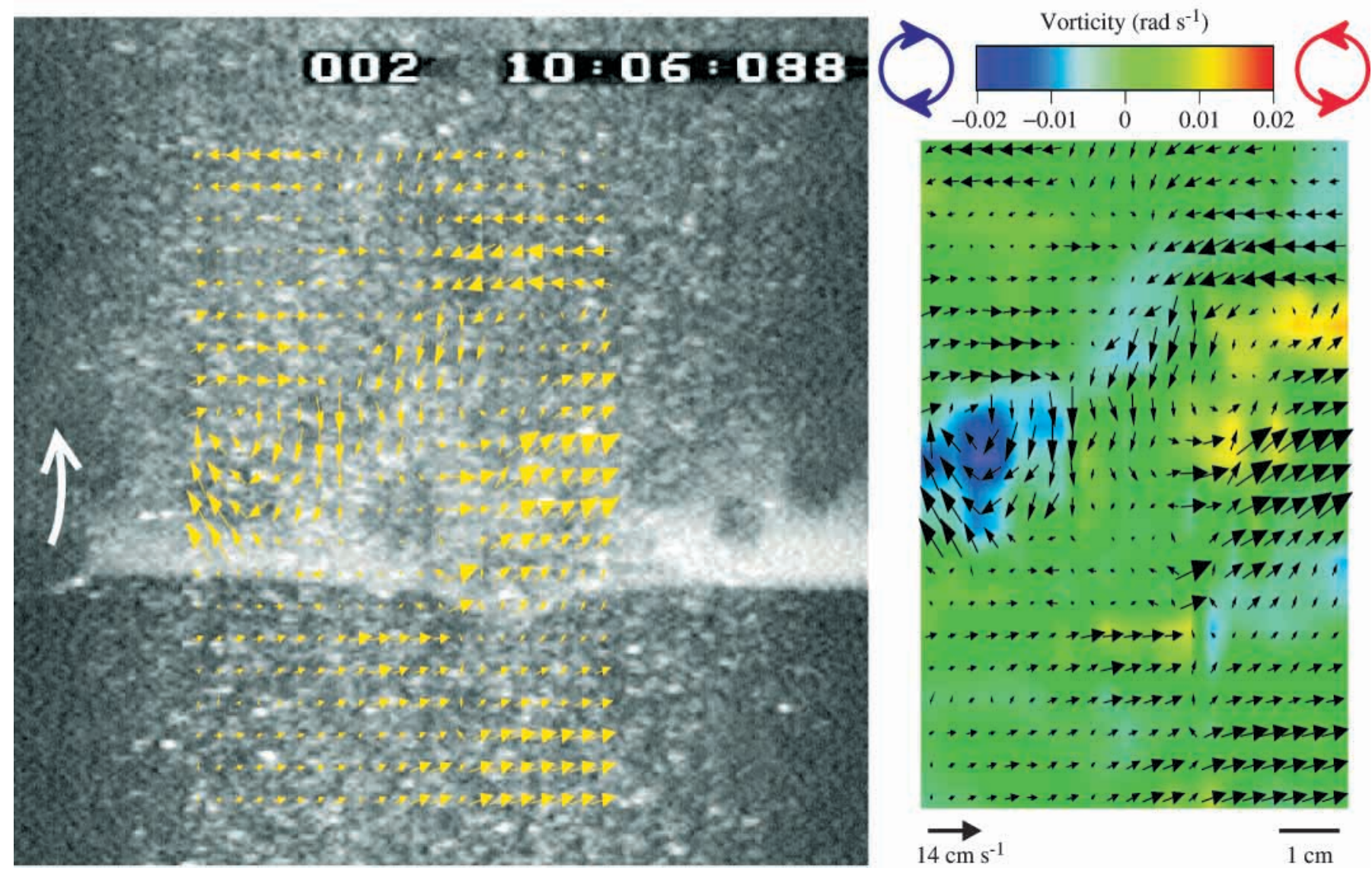

Fig. 9. Digital particle image velocimetry (DPIV) data from leopard shark pectoral fins at the initiation of sinking behavior. Conventions as in Fig. 7. Note that there is a clockwise vortex (blue vorticity on right) which resulted from the upward fin flip (curved white arrow) to initiate the sinking event. The clockwise vortex is indicated by the blue region of rotating fluid. To assist in visualizing the flow pattern, a mean horizontal flow of $U=33 \mathrm{~cm} \mathrm{~s}^{-1}$ was subtracted from each vector. 


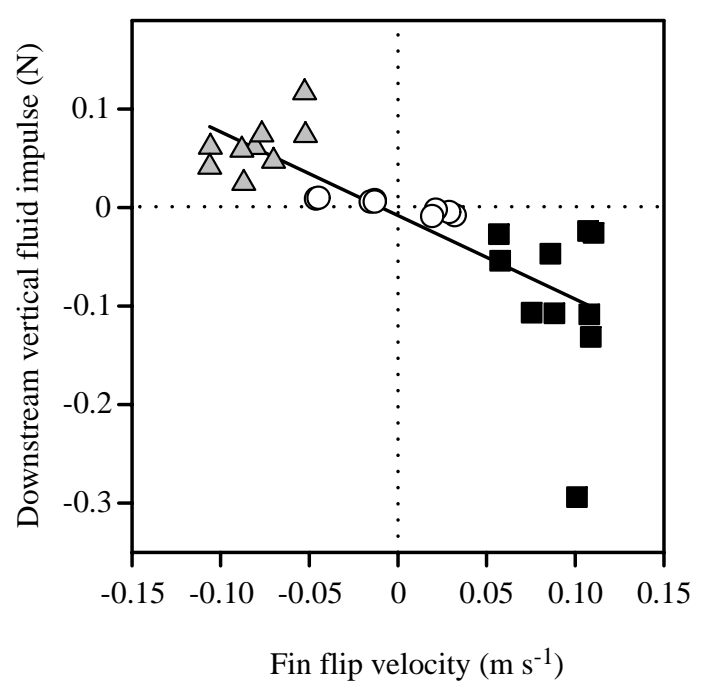

Fig. 10. Plot of the two-dimensional downstream vertical fluid impulse resulting from a pectoral fin flip versus pectoral fin flip velocity. Force was calculated from digital particle image velocimetry (DPIV) measurements (see Materials and methods). Symbols and conventions as in Fig. 3. Negative velocities are defined as those in which the fin moved ventrally, and negative lift forces are oriented downwards. Note that negative lift forces are associated with an upward fin flip (positive velocities), and that greater force results from higher fin flip velocities. The least-squares regression line is significant $\left(P<0.007\right.$; adjusted $\left.r^{2}=0.22\right)$. Downstream vertical fluid impulse is significantly different among the three behaviors (ANOVA, $P=0.01$ ). Values are for 27 DPIV sequences (three fields for three behaviors for three individuals, $N=3$ ).

relative to the anterior plane to produce a mean dorsal obtuse internal fin angle of $185^{\circ}$ (Table 1). At the same time, the leading edge of the fin is rotated downwards relative to the flow such that the chord line is decreased to an angle of attack to the flow of $-22^{\circ}$ (Fig. 6C). As expected, a clockwise vortex is visualized in the wake of the pectoral fin as a result of a dorsal fin flip (Fig. 9).

Lift produced by altering the planar surface of the pectoral fin to rise and sink appears to be a mechanism to reorient the position of the head and anterior body for maneuvering. Changing the orientation of the head will alter the force balance on the body as a result of interaction with the oncoming flow and will induce a change in vertical force that will move the shark up or down in the water column. Forces generated by the pectoral fins are significantly greater in magnitude during sinking than during rising (Table 1). This may be due to the necessity of reorienting the body through a greater angular change to sink from the positive body tilt adopted during steady swimming. Leopard sharks must reposition the body from a positive body tilt of $8^{\circ}$ (mean holding angle) down through the horizontal to a negative body tilt of $-11^{\circ}$ (mean sinking angle), a change of $19^{\circ}$. In contrast, to rise, leopard sharks simply increase the positive tilt of the body by $14^{\circ}$ (mean rise-hold difference), which should require less force given that the oncoming flow will assist the change from a horizontal to a rising body position.

The planar surface of leopard shark pectoral fins is held at a negative dihedral angle of $-23^{\circ}$ relative to the horizontal during steady horizontal swimming (Fig. 11A). The pectoral fins are destabilizing in this position (Smith, 1992; Simons, 1994; Wilga and Lauder, 1999) and promote rolling motions of the body, such as those made while maneuvering in the water column (Fig. 11D). For example, in a roll, the fin with the greatest angle to the horizontal meets the flow at a greater angle of attack, resulting in a greater force $\left(F_{\mathrm{X}}\right)$ directed into the roll, while the angle of attack of the more horizontally oriented fin is reduced by the same amount. This fin therefore possesses a smaller force $\left(F_{\mathrm{X}}\right)$ opposing the roll (Fig. 11D). The resulting horizontal force is greater in the direction of the roll, thereby contributing to the roll. This result is in contrast to previous studies suggesting that the pectoral fins of sharks are oriented to prevent rolling, as in the keel of a ship (Harris, 1936, 1953). Wings that are tilted at a positive angle with respect to the horizontal have a positive dihedral angle (seen in some airplanes) and are stabilizing in that they resist rolling motions of the fuselage (Fig. 11E) (Smith, 1992; Simons, 1994). For example, in a roll, the more horizontally oriented wing generates a greater lift force than the inclined wing, which generates reduced lift (Smith, 1992; Simons, 1994). A corrective restoring moment arises from the imbalance of lift on the two sides, and this imbalance stabilizes the aircraft in roll.

The dihedral angle of leopard shark pectoral fins changes significantly during vertical maneuvering in the water column (Fig. 11B,C). The dihedral angle increases to $-35^{\circ}$ during rising and decreases to $-5^{\circ}$ during sinking. This may be due to a need for greater stability during sinking behavior since the heterocercal tail generates a lift force that tends to drive the head ventrally. Holding the pectoral fins nearly horizontal during sinking would result in increased stability relative to rising. During rising, the increased negative dihedral angle relative to horizontal swimming would increase maneuverability and allow rapid changes in body orientation.

Three-dimensional kinematic analyses of swimming fishes are crucial to deriving accurate hypotheses about the function of the pectoral fins and body (Wilga and Lauder, 1999). Previous studies of swimming sharks modeling the pectoral fin as a two-dimensional rigid flat plate have concluded that the pectoral fins produce lift during steady horizontal swimming (Breder, 1926; Daniel, 1922; Harris, 1936; Aleev, 1969). Indeed, in lateral views of swimming sharks (see Fig. 5B: hold), there appears to be an angle of incidence of approximately $8-10^{\circ}$ between the anterior margin of the fin and flow direction (if fin angle is measured from the anterior fin base to the lateral tip). Quantification of this twodimensional angle during steady horizontal swimming in leopard sharks also resulted in a positive pectoral fin attack angle of $12^{\circ}$, similar to values reported in the literature. It should be noted that this two-dimensional angle as a representation of the planar surface of the pectoral fin in sharks 

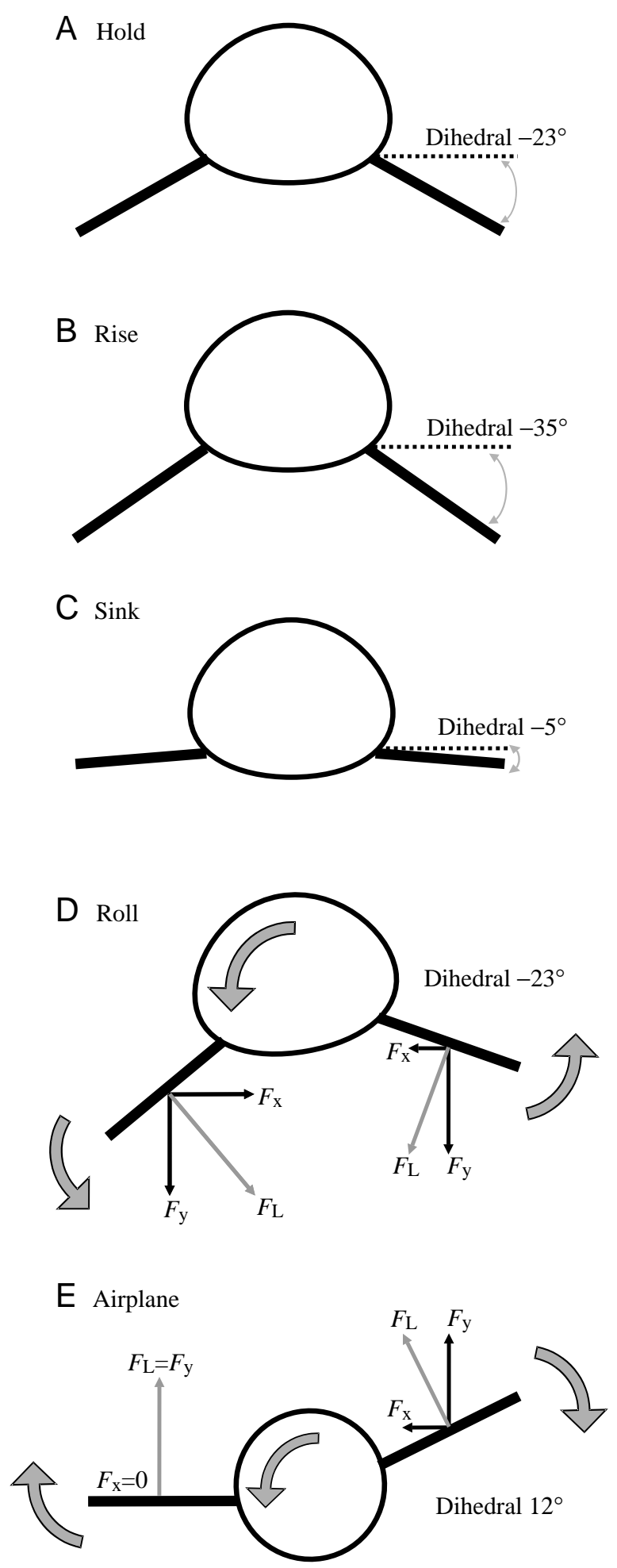

is extremely misleading. Although the pectoral fin appears to be oriented at a positive angle to the flow in lateral view, threedimensional kinematics reveals that the fin is actually concave downwards with a negative dihedral. When viewed laterally, this negative-dihedral concave-downwards orientation of the pectoral fin creates a perspective that suggests a positive angle of attack when the angle is, in fact, negative.
Fig. 11. Schematic diagram of the dihedral orientation of the pectoral fins in a leopard shark during (A) holding, (B) rising and (C) sinking behaviors analyzed in this study. Forces during a roll are illustrated for (D) the pectoral fins of a leopard shark and (E) the wings of an airplane. The body and fin are represented as a cross section at the level of plane $\alpha$ of the pectoral fin (see Fig. 1). Double-headed arrows represent the dihedral angle between the plane $\alpha$ (dotted line) and pectoral fin. Thick arrows show the direction of movement of the body and fins or wing during a roll. Note that positive dihedrals (such as those used in aircraft design, E) are self-stabilizing, while fins oriented at a negative dihedral angle, as in the leopard shark (D), are destabilizing in roll and tend to amplify roll forces. $F_{\mathrm{X}}$, horizontal force; $F_{\mathrm{y}}$, vertical force; $F_{\mathrm{L}}$, resultant force.

\section{Proposed force balance on swimming sharks}

The results of this study on freely swimming leopard sharks in a flow tank are in contrast to previous models of heterocercal tail locomotion of sharks and sturgeon. Classical theory states that the pectoral fins function to generate lift to balance the moments generated by the heterocercal tail (Affleck, 1950; Alexander, 1965; Aleev, 1969; Thomson, 1976; Videler, 1993). The beating of the heterocercal tail produces a lift force that is directed dorsally and anteriorly and deflects water ventrally and posteriorly (Simons, 1970; Ferry and Lauder, 1996). The lift force from the tail then generates a turning moment around the center of balance that tends to pitch the head ventrally. This turning moment is thought to be countered by the passage of dorsally slanted pectoral fins and the ventral surface of the head through the water, which presumably generate a lift force that is directed dorsally. The net upward lift forces are balanced by weight, and the fish are then able to swim in a horizontal plane with both vertical forces and moments in balance.

We propose a new hypothesis for the vertical force balance on leopard sharks during steady horizontal swimming and vertical maneuvering in the water column at $1.0 l \mathrm{~s}^{-1}$ (Fig. 12). The new hypothesis is based on our results showing that the pectoral fins generate negligible lift force during horizontal swimming and on those of Ferry and Lauder (1996) which show that the heterocercal tail of Triakis semifasciata generates upward thrust during steady horizontal swimming. We suggest that four vertical components of force act on leopard sharks during steady horizontal swimming: a downward force at the center of mass, $F_{\text {weight }}$, due to the negative buoyancy of leopard sharks; upward forces due to the dorsally pitched ventral surface of the body cranial to and caudal to the center of mass, $F_{\text {cranial ventral body surface }}$ and $F_{\text {caudal ventral body surface, and an }}$ upward force resulting from oscillation of the caudal fin, $F_{\text {tail }}$ (Fig. 12B). The pectoral fins do not contribute significant lift during steady horizontal swimming. Also, note that the body is tilted at a positive angle of attack to the flow $\left(8^{\circ}\right.$ at $\left.1.0 l \mathrm{~s}^{-1}\right)$. This new force balance hypothesis for steady horizontal swimming suggests that leopard sharks adjust body tilt to balance the forces on the ventral body surface anterior and posterior to the center of mass. The effect of the ventral body surface on the balance of moments depends on the location of 
A Rise
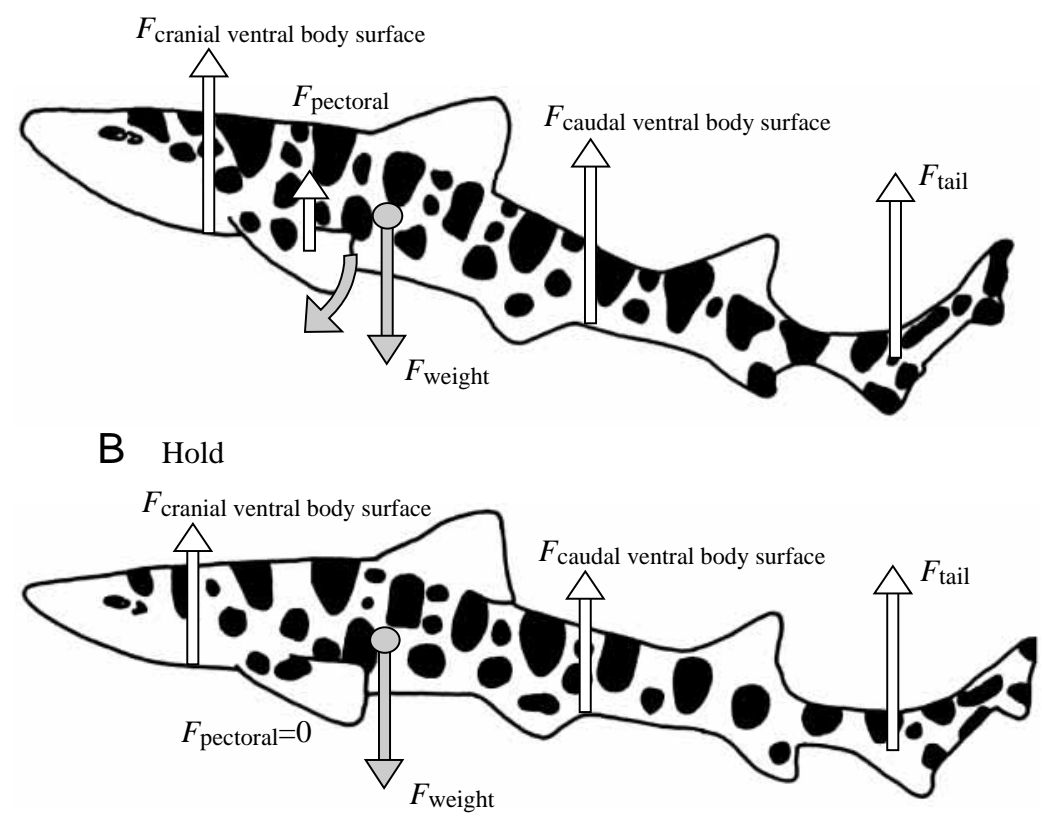

Fig. 12. Diagram of proposed vertical force balance on swimming leopard sharks at $1.0 l \mathrm{~s}^{-1}$, where $l$ is total body length. The gray circle indicates the location of the center of mass, and vectors indicate forces $F$ exerted by the fish on the fluid. In all panels, the tail vector is assumed to generate upward force (see text for discussion) based on the work of Ferry and Lauder (1996). Lift forces are generated by the ventral body surface, both anterior and posterior to the center of mass. (A) Rising; (B) holding position (based on the experimental results of this paper, no forces are generated by the pectoral fins during holding); (C) sinking. The curved arrows indicate the fin flip initiating rising or sinking behaviors.

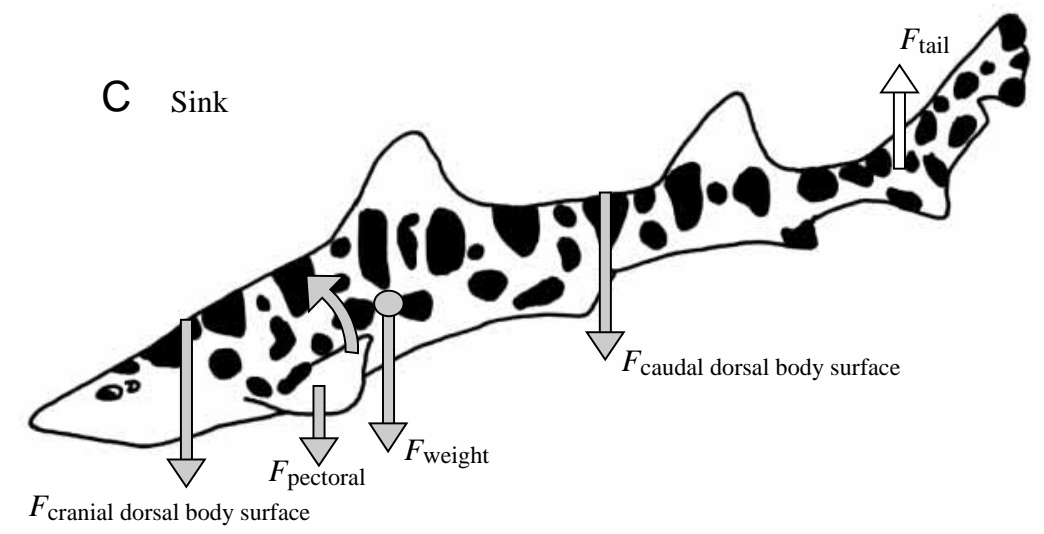

that surface relative to the center of mass. The ventral body surface posterior to the center of mass will generate a moment tending to rotate the head ventrally, while the moment generated by the ventral surface of the head and body anterior to the center of mass will produce a moment rotating the head dorsally.

A fifth vertical lift component due to the pectoral fins, $F_{\text {pectoral}}$, becomes active on leopard sharks during vertical maneuvering in the water column. Ventral rotation of the posterior plane of the pectoral fins at the initiation of rising behavior produces a significant upwardly directed force, which then pitches the anterior region of the body dorsally (Fig. 12A). Thereafter, upward movement of the body is probably effected by the positive tilt of the body interacting with oncoming flow during the remainder of the rising event. Similarly, dorsal rotation of the posterior plane of the pectoral fins at the initiation of sinking behavior generates a significant downward force, which pitches the anterior portion of the body ventrally (Fig. 12C). Again, downward movement of the body is assisted by the negative tilt of the body interacting with oncoming flow for the remainder of the sinking behavior. Thus, the pectoral fins in leopard sharks appear to be critical for initiating maneuvering behaviors in the water column, but not for lift production during steady horizontal swimming.

\section{Comparison of shark and sturgeon pectoral fin function}

The orientation and function of the pectoral fins and body during swimming in leopard sharks are remarkably similar to our previous findings on white sturgeon Acipenser transmontanus (Wilga and Lauder, 1999). Like sturgeon, sharks have an elongate body with a heterocercal tail and a plesiomorphic pectoral fin morphology in which the basals and radials of the fin extend laterally from the trunk. Both leopard sharks and white sturgeon use the ventral body surface to generate lift by adopting a positive body tilt of $8^{\circ}$ to the flow during steady horizontal swimming at $1.0 l \mathrm{~s}^{-1}$ (Table 1). In addition, both fishes adjust total lift by increasing body tilt at 
low flow speeds and decreasing body tilt at higher flow speeds. Furthermore, during vertical maneuvering in both fishes, the positive tilt of the body is increased to rise and decreased to a negative angle to sink. Both leopard sharks and white sturgeon tilt the body into the flow to generate lift and balance moments around the center of mass as appropriate during steady horizontal swimming as well as in vertical maneuvering.

Similarly, the pectoral fins of leopard sharks and white sturgeon are held in a position that suggests that negligible or no lift is generated during steady horizontal swimming. The pectoral fins in both fishes are cambered, concave downwards, with similar dorsal planar angles that are held at a negative chord angle of attack to the flow and are oriented in a negative dihedral (Table 1). DPIV analyses show no evidence of downwash behind the pectoral fins in this orientation during steady horizontal swimming. Thus, in two fishes with heterocercal tails, leopard sharks and white sturgeon, the pectoral fins do not appear to be generating lift.

Vertical maneuvering in the water column is also accomplished similarly in leopard sharks and white sturgeon. Orientation of the pectoral fins during rising is similar in both leopard sharks and white sturgeon (Table 1). As in holding, the pectoral fins are still cambered, concave downwards, with an obtuse dorsal angle and a negative dihedral, but to rise, the posterior plane of the fin is flipped downwards, resulting in a greater positive chord angle to the flow. Similarly, the posterior plane of the fin is flipped upwards to sink, producing a negative chord angle to the flow. Even though the posterior plane of the pectoral fin flips upwards during sinking in leopard sharks, the fin is still cambered concave downwards at a negative dihedral. In contrast, white sturgeon flip the posterior plane of the pectoral fin dorsally to such a degree during sinking that camber is reversed and is now concave upwards with an acute dorsal fin angle of $170^{\circ}$ (Table 1). However, the end result is similar: both leopard sharks and white sturgeon initiate rising or sinking behavior by actively moving the posterior plane of the pectoral fin ventrally or dorsally, respectively, producing a starting vortex that induces a pitching movement reorienting the anterior body in the appropriate direction. Thus, the pectoral fins of leopard sharks and white sturgeon are used to adjust body angle as appropriate to balance moments around the center of mass.

Note that there is at least one important difference in the force balance during steady horizontal swimming in leopard sharks and white sturgeon. Recent studies show that the heterocercal tail of Acipenser transmontanus generates thrust directed near the center of mass (Lauder, 2000; J. Liao and G. V. Lauder, in preparation). In addition, sturgeon, while negatively buoyant, possess a gas bladder. In contrast, the heterocercal tail of Triakis semifasciata generates thrust dorsally and anteriorly to the center of mass (Ferry and Lauder, 1996), and sharks lack a gas bladder. Leopard sharks have to generate more lift anterior to the center of mass than do sturgeon because the tail generates a greater moment tending to rotate the head ventrally. Therefore, both leopard sharks and sturgeon balance moments and lift forces using their ventral body surface, but must necessarily do so in different ways because of differences in the forces generated by the tail.

Are the results presented here applicable to other species of sharks? Do the pectoral fins of other sharks, such as benthic and pelagic species, function primarily for generating forces during maneuvering as in leopard sharks? Or do the pectoral fins of other sharks generate lift during steady horizontal locomotion as predicted by the classical model? Such comparative analyses are the next logical step towards understanding the generality of these results and the functional design of pectoral fins in relation to the diversity of body and tail morphologies exhibited by sharks.

The authors thank two anonymous reviewers for helpful suggestions that improved the manuscript. We also thank Eliot Drucker, Alice Gibb, Stephen Anderson, Lara FerryGraham, Jen Nauen and Ted Stankowich for providing assistance during the experiments and/or analysis. We also thank Eliot Drucker, Alice Gibb, Lara Ferry-Graham, Jimmy Liao, Frank Fish and Jen Nauen for providing helpful comments or discussion during the course of this project. Support was provided by NSF grants to C.D.W. (DBI 9707846) and G.V.L. (IBN 98-07012).

\section{References}

Acheson, D. J. (1990). Elementary Fluid Dynamics. New York: Oxford University Press.

Affleck, R. J. (1950). Some points in the function, development and evolution of the tail in fishes. Proc. Zool. Soc. Lond. 120, 349-368.

Aleev, Y. G. (1969). Function and Gross Morphology in Fish. Translated from the Russian by M. Raveh. Jerusalem: Keter Press.

Alexander, R. McN. (1965). The lift produced by the heterocercal tails of Selachii. J. Exp. Biol. 43, 131-138.

Breder, C. M. (1926). The locomotion of fishes. Zoologica 4, 159-256.

Daniel, J. F. (1922). The Elasmobranch Fishes. Berkeley: University of California Press.

Dickinson, M. H. (1996). Unsteady mechanisms of force generation in aquatic and aerial locomotion. Am. Zool. 36, 537-554.

Drucker, E. G. and Lauder, G. V. (1999). Locomotor forces on a swimming fish: three-dimensional vortex wake dynamics quantified using digital particle image velocimetry. J. Exp. Biol. 202, 2393-2412.

Ferry, L. A. and Lauder, G. V. (1996). Heterocercal tail function in leopard sharks: a three-dimensional kinematic analysis of two models. J. Exp. Biol. 199, 2253-2268.

Fish, F. and Shannahan, D. (2000). The role of the pectoral fins and body trim of sharks. J. Fish Biol. 56, 1062-1073.

Harris, J. E. (1936). The role of the fins in the equilibrium of the swimming fish. I. Wind tunnel tests on a model of Mustelus canis (Mitchell). J. Exp. Biol. 13, 476-493.

Harris, J. E. (1938). The role of the fins in the equilibrium of the swimming fish. II. The role of the pelvic fins. J. Exp. Biol. 16, 32-47.

Harris, J. E. (1953). Fin patterns and mode of life in fishes. In Essays in Marine Biology (ed. S. M. Marshall and A. P. Orr), pp. 17-28. Edinburgh: Oliver \& Boyd.

He, P. and Wardle, C. S. (1986). Tilting behavior of the Atlantic 


\section{C. D. WILGA AND G. V. LAUDER}

mackerel, Scomber scombrus, at low swimming speeds. J. Fish Biol. 29, 223-232.

Hicks, C. R. (1982). Fundamental Concepts in the Design of Experiments, 3rd edition. Orlando: Harcourt Brace Jovanovich College Publishers.

Jayne, B. C. and Lauder, G. V. (1995). Are muscle fibers within fish myotomes activated synchronously? Patterns of recruitment within deep myomeric musculature during swimming in largemouth bass. J. Exp. Biol. 198, 805-815.

Jayne, B. C., Lozada, A. and Lauder, G. V. (1996). Function of the dorsal fin in bluegill sunfish: motor patterns during four locomotor behaviors. J. Morph. 228, 307-326.

Johnson, C. S. (1970). Some hydrodynamic measurements on sharks. Nav. Underwater Cent. Tech. Rep. 189, 13pp.

Klausewitz, W. (1962). Wie schimmen Haifische? Nat. Mus. Frankf. 92, 219-226.

Klausewitz, W. (1965). Die Bewungegsweise der Geigenrochen aus functioneller und stammesgeshichtlicher Sicht. Nat. Mus. Frankf. 95, 97-108.

Krothapalli, A., Lourenco, L. and Shih, C. (1997). Visualization of velocity and vorticity fields. In Atlas of Visualization III (ed. Y. Nakayama and Y. Tanida), pp. 69-82. Boca Raton, FL: CRC Press.

Kundu, P. (1990). Fluid Mechanics. San Diego: Academic Press.

Lauder, G. V. (1989). Caudal fin locomotion in ray-finned fishes: historical and functional analyses. Am. Zool. 29, 85-102.

Lauder, G. V. (2000). Function of the caudal fin during locomotion in fishes: kinematics, flow visualization and evolutionary patterns. Am. Zool. 401, 101-122.

Lauder, G. V. and Jayne, B. C. (1996). Pectoral fin locomotion in fishes: testing drag-based models using three-dimensional kinematics. Am. Zool. 36, 567-581.

Prandtl, L. and Tietjens, O. G. (1934). Applied Hydro- and
Aeromechanics. Translated by J. P. Den Hartog. New York: Dover Publications, Inc.

Raffel, M., Willert, C. and Kompenhans, J. (1998). Particle Image Velocimetry: A Practical Guide. Heidelberg: Springer-Verlag.

SAS (1998). SAS/STAT System for Personal Computers. Cary, SAS Institute Inc.

Simons, J. R. (1970). The direction of the thrust produced by the heterocercal tails of two dissimilar elasmobranchs: the Port Jackson shark, Heterodontus portusjacksoni (Meyer) and the piked dogfish, Squalus megalops (Macleay). J. Exp. Biol. 52, 95-107.

Simons, M. (1994). Model Aircraft Aerodynamics. Herts, UK: Argus Books.

Smith, H. C. (1992). The Illustrated Guide to Aerodynamics. New York: TAB Books.

Thomson, K. S. (1976). On the heterocercal tail in sharks. Paleobiol. 2, 19-38.

Thomson, K. S. and Simanek, D. E. (1977). Body form and locomotion in sharks. Am. Zool. 17, 343-354.

Videler, J. J. (1993). Fish Swimming. New York: Chapman \& Hall.

Vogel, S. and Feder, N. (1966). Visualization of low-speed flow using suspended plastic particles. Nature 209, 186-187.

Webb, P. W. (1993). The effect of solid and porous channel walls on steady swimming of steelhead trout Oncorhynchus mykiss. J. Exp. Biol. 178, 97-108.

Webb, P. W. and Keyes, R. S. (1982). Swimming kinematics of sharks. Fish. Bull. 80, 803-812.

Wilga, C. D. and Lauder, G. V. (1999). Locomotion in sturgeon: function of the pectoral fins. J. Exp. Biol. 202, 2413-2432.

Willert, C. E. and Gharib, M. (1991). Digital particle image velocimetry. Exps Fluids 10, 181-193.

Zar, J. H. (1996). Biostatistical Analysis, 3rd edition. Upper Saddle River, NJ: Prentice Hall. 